\title{
Finite mixture distribution models of simple discrimination learning
}

\author{
MAARTJE E. J. RAIJMAKERS, CONOR V. DOLAN, and PETER C. M. MOLENAAR \\ University of Amsterdam, Amsterdam, The Netherlands
}

\begin{abstract}
Through the application of finite mixture distribution models, we investigated the existence of distinct modes of behavior in learning a simple discrimination. The data were obtained in a repeated measures study in which subjects aged 6 to 10 years carried out a simple discrimination learning task. In contrast to distribution models of exclusively rational learners or exclusively incremental learners, a mixture distribution model of rational learners and slow learners was found to fit the data of all measurement occasions and all age groups. Hence, the finite mixture distribution analysis provides strong support for the existence of distinct modes of learning behavior. The results of a second experiment support this conclusion by crossvalidation of the models that fit the data of the first experiment. The effect of verbally labeling the values on the relevant stimulus dimension and the consistency of behavior over measurement occasions are related to the mixture model estimates.
\end{abstract}

\section{Introduction}

The existence of distinct modes of learning, such as implicit learning and explicit learning, though often posited, is not easy to establish empirically. In the present article, we propose using the statistical technique of finite mixture distribution modeling (Everitt \& Hand, 1981) to investigate the existence of different modes of learning a simple discrimination during development. The main purpose of the application of this technique is to establish whether multiple learning modes exist during development.

One generally accepted taxonomy of learning systems concerns learning by focusing on particular items versus learning by focusing on the underlying rules. Stadler (1997) argues that the distinction between implicit and explicit learning should be viewed as a distinction between intentional and unintentional learning. Intentional learning involves focusing on rules, whereas unintentional learning involves focusing on items. Furthermore, Dienes and Berry (1997) stated that "implicit learning tends to be associated with observation and memorization conditions, rather than deliberate hypothesis testing" (p. 5). Shanks and St. John (1994), who questioned the existence of an implicit learning system that involves learning without concurrent awareness, concluded that "the distinction

The Netherlands Organization for Scientific Research (NWO) is gratefully acknowledged for funding this project. This research was conducted while M.E.J.R. was supported by Grant 575-21-005 of the Foundation for Behavioral and Educational Sciences. The research of C.V.D. was made possible by a fellowship of the Royal Netherlands Academy of the Arts and Sciences. We thank Han van der Maas for helpful comments on earlier drafts of this article. In addition, we acknowledge the helpful comments of the reviewers of earlier drafts of this article. Correspondence concerning this article should be addressed to M. E. J. Raijmakers, Department of Developmental Psychology, University of Amsterdam, Roetersstraat 15, 1018 WB Amsterdam, The Netherlands (e-mail: op_raijmakers@ macmail.psy.uva.nl). between instance and rule learning is a sound and meaningful way of taxonomizing human learning" (p. 367).

A generally accepted way to establish distinct modes of learning is to show that a given experimental manipulation (e.g., a speeded test; Turner \& Fischler, 1993) has a different, preferably opposite, effect on the learning behavior of subjects who learn explicitly and on the learning behavior of subjects who learn implicitly (cf. Neal \& Hesketh, 1997; Shanks \& St. John, 1994; Stadler, 1997, provide reviews of these approaches). This method of establishing differences between learning modes is based on the idea that learning processes can be distinguished if they are affected differently by the same experimental manipulation.

In the present article, we follow a different approach in establishing the distinctiveness of learning modes. Instead of searching for distinguishing properties of distinct modes of learning, we attempted to establish whether there are distinct modes of learning or a single general mode of learning with a continuum of appearances. Given the existence of distinct modes of learning, focusing on items could represent one mode, and focusing on rules could represent the other. In this case, each learning event can be completely characterized by a single given mode. If, on the contrary, there is one general mode of learning with a continuum of manifestations, focusing on rules could represent one pole and focusing on items the other. According to Reber (1997), the distinction between implicit learning and explicit learning (which he characterizes mainly as unconscious and conscious processes, respectively) is not between two isolated cognitive modules but between two poles on a continuum. In contrast, Ashby, Alfonso-Reese, Turken, and Waldron (1998) posit the existence of two separate categorization systems, one verbal and one implicit. Both learning systems operate in parallel within an individual, but one system may 
dominate the other depending on the individual, the task, and the environment. For example, according to Ashby et al. the verbal system in young children is not fully developed. Hence, Ashby et al.'s model predicts that young children are at a disadvantage in category learning tasks in which the optimal rule is verbal. Furthermore, this model predicts that the categorization system that is actually applied by a subject in a categorization task can be manipulated experimentally. Both predictions are addressed in the present article.

In developmental psychology, bimodality of the distribution of scores has traditionally been considered a necessary condition for the existence of distinct modes of behavior (e.g., Fischer, Pipp, \& Bullock, 1984; van der Maas \& Molenaar, 1992; Wohlwill, 1973). An appropriate statistical technique to investigate the modality of scores is finite mixture distribution modeling (Everitt \& Hand, 1981; Titterington, Smith, \& Makov 1985). Finite mixture distribution modeling may be used to investigate whether a distribution of test scores is a mixture of multiple component distributions. A distribution is expected to be bimodal if the data follow a two-component mixture distribution, and the means of the two component distributions are sufficiently separated.

In the present article, our main aim was to investigate whether distinct modes of simple discrimination learning exist during development. Kendler (1979) has already presented some evidence in support of the existence of two learning modes, which she calls rational learning and incremental learning. However, because Kendler (1979) did not apply entirely appropriate methodological techniques, the existence of distinct modes of discrimination learning has yet to be established. Here, we apply the statistical technique of finite mixture distribution modeling to cross-sectional data obtained in a simple discrimination learning task. A simple discrimination is one with a single relevant stimulus dimension. Relevance is defined with respect to the reinforcement contingencies. A large body of empirical studies shows that adults learn a simple discrimination by hypothesis testing of rules (e.g., Gohlson, Levine, \& Phillips, 1972). In contrast, children under 6 years of age generally do not apply rules systematically and consequently learn less efficiently (Kendler, 1979). We investigate whether the individual differences in the number of errors give rise to a two-component distribution of performance. In this mixture, one component represents the distribution of errors committed by subjects who learn by hypothesis testing. The other component represents the distribution of errors committed by subjects who learn slowly. This is a special case of a distribution derived from a single-operator linear model. Henceforth, we refer to these types of learning as rational learning and slow learning, respectively. If individual differences in errors are found to follow a two-component distribution, this would be good evidence in support of the existence of two modes of simple discrimination learning during development (rational learning and slow learning, which is consistent with incremental learning) and the absence of intermediate learning behavior. A continuous range of manifest behavior of the learning system is not compatible with a two-component mixture distribution of errors, as considered here.

If a two-component mixture distribution is found to be tenable in a cross-sectional sample, the distribution of the members of each component over age groups should be examined. The question is whether the two-component structure of the data is attributable to the age structure of the sample, so that component membership is directly related to age. In that case, the two-component structure of the data could be an artifact of the age structure of the sample. Furthermore, age-related differences can be expressed in terms of the finite mixture distribution model. For example, the relative size of each component may be related to age, which would indicate that the proportion of subjects who learn rationally is related to age.

A two-component mixture implies that at any instance in time an individual learns either rationally or slowly, but does not display any form of intermediate behavior. However, an individual does not necessarily apply one and the same learning mode at each occasion. For example, an individual need not necessarily learn incrementally until a given age (which may differ from individual to individual) and then shift irrevocably to the rational learning mode. According to Ashby et al. (1998) two learning systems exist in parallel within an individual. At different moments in time, subjects may apply different learning systems. For example, learning by children, in whom the verbal learning system is not fully developed, may occasionally be dominated by the implicit learning system. We examined the subjects' consistency in their application of a learning mode by means of a repeated measures design. We examined the robustness of the two learning modes by including an additional experimental manipulation involving verbal labeling. Verbal labeling of the values on the relevant stimulus dimension during learning is believed to encourage subjects to learn the discrimination rationally (Ashby et al., 1998; Kendler, 1995). Nevertheless, if the two learning modes are robust, this manipulation is expected to result in a model consisting of the same two components observed in the standard condition of the task. The manipulation is expected to affect only the number of subjects that belong to the rational component.

Finite mixture distribution modeling is closely related to Siegler's (1981) rule-assessment methodology for analyzing individual differences in terms of strategies. $\mathrm{La}$ tent class analysis, which is a finite mixture distribution modeling technique (Wolfe, 1970), is a statistical formalization of the rule-assessment methodology that makes it possible to test statistically the number and nature of the rules that children apply (Jansen \& van der Maas, 1997). We hope that the present article will show the benefits of the finite mixture modeling technique in the analysis of individual differences in simple discrimination learning. In addition to the analyses of the discrimination data, we consider in detail certain aspects of the finite mixture distribution methodology relating to power analysis and Monte Carlo bootstrap of test statistics. 
1.1. Overview. In the next section, we discuss Kendler's (1979) evidence for the existence of distinct modes of simple discrimination learning. In the third section, we describe the design of the task and the experiment that we used to assess discrimination learning. Then, in the fourth section, we present the models that give rise to the component distributions of the proposed finite mixture distribution. The model of rational learning is based on the Markov model of concept identification (Bower \& Trabasso, 1964). The model of incremental learning is a single-operator linear model of incremental learning (Estes, 1950). To identify the simplest, best fitting model, a series of models is fitted to the data. In the fifth section, we explain our approach to the methods of model selection. Model selection is based on the likelihood ratio goodness-of-fit statistic and on the Akaike's information criterion. We perform power analyses to show how well the competing models can be distinguished given the available sample sizes. In the six th section, we present results of the data analyses. We first carry out nonparametric tests to investigate differences between conditions and groups. The results of these preliminary tests are related to results obtained by fitting the mixture distribution models. Then we calculate maximum likelihood estimates of the parameters of the various mixture models. We analyze the data in two ways: by measurement occasion and by age group. Furthermore, we examine the consistency of the application of a learning mode over repeated measures. To this end, at each measurement occasion, we assigned individuals to one of the components on the basis of Bayesian posterior probabilities. In a second experiment, discussed in Section 7, we cross-validate the models that were obtained in the first experiment. Finally, we discuss the benefits of our approach and the implications of the results for neural network modeling of discrimination learning, which is currently the main paradigm in modeling learning mechanisms.

\section{Kendler's Evidence for a Two-Component Distribution}

Within the developmental study of discrimination learning, the existence of distinct modes of behavior is an important issue. For example, Kendler's (1995) theory concerning levels of cognitive development posits the existence of two modes of executive behavior and two modes of encoding behavior. Executive behavior, which we simply call learning, is either rational or incremental. Rational learning is based on hypothesis testing. Incremental learning is based on the gradual strengthening of stimulus-response relations. Alternatively, Zeaman and House (1963) suggested that developmental differences of discrimination learning are due to different values of parameters, such as learning rate and extinction rate, in one and the same model. They proposed an attention model of discrimination learning (e.g., Zeaman \& House, 1974b). Kendler (1979) has presented evidence in support of the hypothesis that the distribution of test scores, which are related to executive behavior, is a mixture of two distributions. However, because appropriate techniques were not applied, the existence of distinct modes of executive behavior has yet to be established conclusively.

The efficiency with which a discrimination task is solved increases with age. Kendler (1979) showed that, in a simple discrimination task with four stimuli differing on two dimensions, the number of errors committed before a given criterion is reached is described well by the following power function of age: $y=a x^{-b}$, where $x$ is age and $a$ and $b$ are parameters. The parameter values of this power function depend on the dimensions of the stimuli. According to Kendler (1979), the gradual increase of efficiency with age is due to an increase of the proportion of children who learn rationally instead of incrementally. Since Kendler (1979) hypothesized two distinct modes of behavior, she expected a two-component mixture distribution of errors.

Kendler's (1979) evidence in support of the twocomponent mixture distribution of the number of errors is based on the following analysis. She first established the proportion of rational learners by counting the number of subjects who followed the win-stay rule during a different discrimination task involving only two distinct stimuli. The win-stay rule implies that errors are no longer committed once a correct response has been given. Then, on the basis of a Markov model, she calculated the expected distribution of the errors of the rational learners. Her Markov model is a modified concept-identification model (Bower \& Trabasso, 1964). Next, she subtracted the expected distribution of errors of the rational learners from the observed distribution of errors. The remaining distribution of the logarithm of the number of errors was found to fit a normal distribution. This distribution was presumed to be the distribution of errors of the incremental learners. As a test for the existence of distinct modes of behavior, Kendler's (1979) procedure is inconclusive. First, the proportion of rational learners was established indirectly. The correlation between the subjects' number of errors in the four-stimuli discrimination task and their classification as rational or incremental learners by the win-stay rule was not established. Second, the fit of a mixture model was not compared with the fit of alternative (e.g., simpler) models. Third, there was no theoretical underpinning for the finding that the distribution of the logarithm of the number of errors of incremental learners is normal. According to Kendler (1979), the approximate normal distribution of errors is plausible if the learning rate of incremental learners is normally distributed and the logarithm of the number of errors reflects the learning rate. The method of finite mixture distribution modeling overcomes most of the limitations of Kendler's (1979) method.

\section{Assessing Simple Discrimination Learning}

3.1. Subjects. The present sample consisted of 71 children from second year kindergarten to fourth year primary school at a school in The Netherlands. ${ }^{1}$ The age range was 6 to 10 years, with an average age of 7 years, 7 months. The sample consisted of 32 girls and 39 boys.

3.2. Materials and Procedure. We assessed the learning behavior on a simple two-choice discrimination learn- 
ing task. ${ }^{2}$ Stimuli that differed on two dimensions were presented on a computer screen in pairs. The two dimensions were shape (either a circle or a triangle) and size (big or small). ${ }^{3}$ In addition, the position of the stimuli on the screen varied (left or right), but position was not relevant to the discrimination of the stimuli. The stimulus pairs in the sequence were randomized in groups of four. The subjects responded by choosing either the left or the right stimulus. This choice was indicated by pressing a marked key on the left or the right of a keyboard. Reinforcement consisted of either a cross (negative) or a smile (positive) that appeared for $2 \mathrm{sec}$ under the chosen stimulus, directly after responding. After $2 \mathrm{sec}$, the stimuli and the reinforcement signal disappeared. The process of learning to discriminate was continued until the criterion was reached that 9 of the last 10 trials were correct. The discrimination task comprised a maximum of 100 trials, after which the test was terminated.

Before the actual test started, the subjects were instructed during training trials. The training trials included stimulus dimensions that differed from those used in the actual test. In the training trials, the stimulus dimensions were the number of squares (one or two) and the design of the squares (plain gray or black stripes). The correspondence between the marked keys on the keyboard and the stimuli was explained to the subjects. The subjects were then required to complete three training trials. During these trials, the manner of reinforcement (smile or cross) was explained and demonstrated. Before the actual test started, the subjects were told that they were going to play a game in which they had to identify and point out the correct pictures.

To establish the consistency of the subjects' responses over measurements, we conducted the test at three repeated measurements (A to C) during two weeks. On an additional measurement occasion (D), the subjects were required to supply verbal labels for the values on the relevant dimension. The subjects were not told that the stimulus dimension that they were required to label was relevant to the discrimination. The relevant stimulus dimension and correct cue were counterbalanced over all measurements and conditions, within and between subjects.

\section{Finite Mixture \\ Distribution Models of Learning}

We investigated the hypothesis of the existence of distinct modes of learning behavior by fitting finite mixture distribution models to cross-sectional data. With this methodology, we model distributions of task performance. Here, we fit mixture distribution models to the distribution of the errors committed during the first $n$ trials of learning a simple discrimination. Modeling the errors observed at all trials was not feasible due to the large number of empty or nearly empty cells in the expected distribution. Although the actual choice of $n$ is arbitrary, we set $n$ to equal 16 , so that we had 17 cells. We provide the motivation for this choice below. A finite mixture distribution is composed of a number of distinct components
(Everitt \& Hand, 1981; Titterington et al., 1985). The present finite mixture distribution model is a mixture of two component distributions. One component represents the distribution of errors of rational learners, and the other component is a null model of the distribution of errors of slow learners. This null model is a special case of the distribution of errors of incremental learners. For each component, we specify a distribution function. To model the distribution of a mixed group of learners, we do not need to know a priori whether a given case is a rational learner or a slow learner. In the more technical terms of the mixture distribution, we need not know the case's component membership. We do have to estimate the proportion of rational and slow learners. To determine whether the sample of learners actually consists of two groups, the fit of the two-component mixture distribution model is compared to the fit of a one-component model of rational learners and a one-component model of incremental learners.

In the present application of finite mixture modeling, the aim is to determine the number and the type of components in the mixture, to estimate the unknown parameters of the distribution functions, and to assign the cases to their respective components on the basis of a posteriori probabilities. Applications of this technique to developmental studies can be found in Thomas and Turner (1991; see also Thomas \& Lohaus, 1993), Hosenfeld, van der Maas, and van den Boom (1997a, 1997b), and Raijmakers, van Koten, and Molenaar (1996). Extensions of the technique to, for example, structural equation modeling of multivariate normal data have been made (e.g., Dolan $\&$ van der Maas, 1998).

4.1. Rational Learning. We derive the probability mass function of the number of errors of rational learners from a Markov model of hypothesis testing: the TrabassoBower concept-identification ( $c i$ ) model (Bower \& Trabasso, 1964). To perform a simple discrimination, a rational learner attends to one stimulus dimension. The stimuli of the present task have two discriminating stimulus dimensions, shape and size. The shape was either a circle or a square; the size was either big or small. Therefore, there were four different cues and so four possible hypotheses concerning the nature of the relevant stimulus dimension. A subject learns by testing these hypotheses until the correct hypothesis is found. According to the $c i$ model, learning a simple discrimination takes place as follows: A subject chooses randomly a hypothesis from the set of all possible hypotheses and applies the chosen hypothesis at each trial until an error is committed. After committing an error, the subject chooses randomly a hypothesis from the subset of all the hypotheses that predicts the occurrence of the last error. This subset includes the hypotheses that were chosen at trials previous to the last trial. Once the subject has selected the correct hypothesis, he or she no longer commits errors and hence will continue to base responses on this hypothesis.

The $c i$ model is a first-order Markov model. This means that trials in a sequence generated by the Markov process 
are dependent, so that the outcome of trial $j+1$ is directly dependent on the outcome of trial $j$, but conditionally independent of the outcomes of trials prior to trial $j$. The $c i$ model has two states: the presolution state and the solution state. In the presolution state, a subject applies an incorrect hypothesis concerning the nature of the relevant stimulus dimension. An incorrect hypothesis results in an error on $50 \%$ of the stimuli, because each combination of stimulus features appears equally often. Consequently, the response of a subject in the presolution state is correct half of the time. Hence, the probability of committing an error in the presolution state, that is, $q$, equals .5 according to the design of the task. Responding in the presolution state is a Bernoulli random process with independent trials (see the transition matrix in the Appendix). Learning according to the $c i$ model consists of making a transition from the presolution state to the solution state. In the solution state, a subject attends to the relevant stimulus dimension and consistently responds correctly. Once a subject has selected the correct hypothesis, he or she will continue to base responses on this hypothesis. Therefore the solution state is a so-called absorbing state.

An important assumption of the $c i$ model is that a subject only switches hypotheses if he or she commits an error. The probability of selecting the correct hypothesis after making an error is $c$. We refer to this solution probability per error as the learning rate for the problem. With probability $1-c$, a subject stays in the presolution state. The probability $c$ is related to the number of stimulus dimensions and to the number of values on each stimulus dimension. In the present task, there were four different cues and hence four possible hypotheses. $c$ is the probability of selecting the correct hypothesis after committing an error. This is one divided by the number of hypotheses that predict the last error. Since half of all possible hypotheses, which equals two here, are consistent with the last error, $c=1 / 2$. However, since we simultaneously present two stimuli from which a subject chooses, position of the stimulus (right or left) might also be considered a stimulus dimension. In that case, $c$ equals $1 / 3$. Hence, for the present task, $c$ is expected to lie between .33 and .5. To calculate the probability of committing an error on each trial, one also needs to know the probability of starting in the solution state at Trial 1 (cf. Kendler, 1979). This is the probability of selecting the correct hypothesis without any prior knowledge. As many different hypotheses exist as there are possible cues. In the first case (without considering position as a stimulus dimension), there are four possible cues and, in the second case, there are six possible cues. Hence the probability of starting in the solution state equals $1 / 4$ or $1 / 6$ - that is, $c / 2$.

Undoubtedly, the $c i$ model is a rough approximation of a presumably wide spectrum of hypothesis testing behavior (cf. Gohlson et al., 1972). For example, Levine (M. Levine, Miller, \& Steynmeyer, 1967) distinguished between several procedures for hypothesis testing that differ in efficiency. In the case of focusing, the most efficient procedure, responses are based on all former tri- als and not only on the penultimate trial. Unfortunately, with the application of finite mixture distribution models, the power of the goodness-of-fit tests is too low to discriminate between different forms of hypothesis testing, in addition to discriminating between slow learning and hypotheses testing (see the section on power analysis). Moreover, our main objective was to discriminate between rational learners and slow learners and not to discriminate between different kinds of rational learners. Hence we chose one simple model of hypothesis testing, the $c i$ model. Kendler (1979) showed that this model fits the distribution of errors reasonably well in a group of adults.

We derive the probability mass function of the number of errors in the first $n$ trials. That is, we only look at the first $n$ trials that a subject encounters, and, in these $n$ trials, we count the number of errors. If a subject reaches criterion before the $n$th trial, we count the total number of errors in the trials he or she completed. To fit the model on the data, we fix $n$ at a particular value. $T_{n}$ is the random variable of interest. For case $i, 1 \leq i \leq N$, with $N$ being the number of cases, $T_{n}$ is defined as follows:

$$
T_{n}=\sum_{j=1}^{n} Y_{j},
$$

$Y_{j}=1$ if $i$ makes an error at trial $j, Y_{j}=0$ otherwise.

The Appendix contains the derivation of the probability mass function of $T_{n}$ from the $c i$ model. Equation $1 \mathrm{de}$ fines the probability mass function of $T_{n}$ for rational learners as

$$
\begin{aligned}
\operatorname{ci}(x ; c, q, n)= & \operatorname{Pr}\left(T_{n}=0\right)=\frac{c}{2}+\left(1-\frac{c}{2}\right)(1-q)^{n} \\
\operatorname{Pr}\left(T_{n}=x\right)= & \left(1-\frac{c}{2}\right) q c[q(1-c)]^{x-1} \sum_{j=x}^{n}\left(\begin{array}{c}
j-1 \\
x-1
\end{array}\right)(1-q)^{j-x} \\
& +\left(1-\frac{c}{2}\right)\left(\begin{array}{l}
n \\
x
\end{array}\right)[q(1-c)]^{x}(1-q)^{n-x}
\end{aligned}
$$

$1 \leq x \leq n$

The parameter $c$ is the learning parameter. The parameter $q$ is the conditional probability of making an error in the presolution state, which is fixed at .5. Hence, to fit the $c i$-distribution model to the data, only the parameter $c$ is estimated. The parameter $c$ is expected to lie between .33 and .5 .

4.2. Incremental and Slow Learning. To model incremental learners, we apply the single-operator linear ( $\mathrm{sol}$ ) model of Estes (1950). According to the sol model, the probability of committing an error decreases continuously with trial number but is independent of the outcome of preceding trials. Therefore, the sol model is a simple, zero-order incremental learning model. The zero-order structure of the model allows for derivation of the distribution of the number of errors in the first $n$ trials. The statistical tests for Markov chains will show that it is not necessary to apply a more complicated, higher-order 
model of incremental learning, such as the model of learning by small steps (Norman, 1972).

The sol model satisfies a difference equation that relates the probability of an error on trial $j+1, P_{j+1}$, to that on Trial $j, P_{j}$. The equation is as follows:

$$
P_{j+1}=P_{j}(1-\alpha)
$$

where $\alpha$ is the learning rate parameter. The solution of this equation is a power function of the form

$$
P_{j}=P_{1}(1-\alpha)^{j-1} .
$$

By design of the task, $P_{1}$ equals .5 . To obtain the probability mass function of the number of errors in the first $n$ trials, one must consider the joint probabilities of all $65,536\left(2^{12}\right)$ sequences of correct responses and errors in the first $n$ trials. The sum of these joint probabilities for all sequences in which the number of errors is exactly $x$ gives $\operatorname{Pr}\left(T_{n}=x\right)$ for the sol model. Because of the length of the resulting expressions, which we derived in Maple (Heck, 1997), we do not reproduce them here. In order to model the frequency data using this function, we fix $n$ at a particular value, and we need only estimate the parameter $\alpha$

$$
\operatorname{sol}(x ; t, \alpha, n)=\operatorname{Pr}\left(T_{n}=x\right) .
$$

We apply this function as a one-component singleoperator linear (sol-) model to test whether the data can be described by a model of incremental learners only. In the mixture distribution model consisting of two components, we apply a simplification of this model: We fix the learning parameter, $\alpha$, to equal zero. That is, we assume that, during the first $n$ trials, the incremental learners do not learn significantly. It is important to note that, eventually, the incremental learners did master the discrimination task, because only subjects who reached the stopping criterion within 100 learning trials are included in the analyses. Note that if the two-component model with the simplified sol component fits the data, a two-component model with a sol component will fit as well or better. The simplified sol model is a null model of any kind of slow learning, including, for example, learning by small steps (Norman, 1972), but it is also a model of no learning. Because in the present application we only model subjects who eventually learned the task, we refer to this model as a model of slow learning.

Fixing $\alpha$ to zero implies that the probability of committing an error is independent of the trial number during the first $n$ trials. In this case, by the design of the task, on each trial, a slow learner makes an error with a probability $t$, which equals .5. Hence, according to this null model, the distribution of errors in the first $n$ trials is a binomial distribution. In a two-component model, we model the component distribution of the number of errors in the first $n$ trials of slow learners by a binomial distribution with mean $(n t)$. Again, $T_{n}$, as defined above, is the variable of interest. Now, $Y_{j}$ is a Bernoulli random variable taking on values $(0,1)$ with a probability $t$. The binomial distribution of the random variable $T_{n}$ is defined as follows:

$$
b(x ; t, n)=\operatorname{Pr}\left(T_{n}=x\right)=\left(\begin{array}{l}
n \\
x
\end{array}\right) t^{x}(1-t)^{n-x}, 0 \leq x \leq n .
$$

If, in contrast to our assumptions, incremental learners do learn during the first $n$ trials, a binomial distribution would not be an appropriate model of their distribution of errors. We tested our assumption in two ways. First, if the probability of committing an error decreases during the first $n$ trials, the probability of committing an error is not stationary. Therefore, in the results section, we first test the stationarity of the first $n$ trials. A second test concerns the estimated value of parameter $t$ from the data. The assumption that incremental learners do not learn during the first $n$ trials is only true if their probability of making an error equals .5. In the results section, we test whether the estimate of parameter $t$ significantly deviates from .5 .

4.3. A Mixture Model of Rational and Slow Learners. A mixed population of rational learners and slow learners is modeled by a finite mixture distribution model composed of the two component distributions, defined in Equations 1 and 3. The probability mass function of the two-component mixture is defined as:

$f(x ; t, c, q, \pi, n)=\pi b(x ; t, n)$

$$
+(1-\pi) \operatorname{ci}(x ; c, q, n), 0 \leq x \leq n,
$$

where $\pi$ is the mixing proportion that equals the proportion of cases that are slow learners. Its complement, 1 $\pi$, equals the proportion of cases that are rational learners. The two-component mixture has five parameters of which $n$ and $q$ are fixed to a priori values. Parameter $q$ is fixed at .5 , as explained above, and $n$ is fixed at 16 . The choice of fixing $n$ at 16 has both advantages and disadvantages. Analyzing fewer trials would result in more reliable goodness-of-fit statistics (see the section on goodness-of-fit statistics). A second advantage of a smaller $n$ is that the assumption about slow learners (i.e., that slow learners do not learn considerably during the first $n$ trials) becomes less strong. However, a drawback of decreasing $n$ concerns a relation between $n$ and the separation between the two components, given the hypothesized values of the parameters (i.e., $q=.5, c=.5$ or $.33, t=.5)$. Specifically, as $n$ increases, the two components become better separated. For the calculation of posterior probabilities of cases belonging to a component (see the next section), a sufficient separation of components is important. Hence, we chose $n$ to be 16 .

In fitting this model to the data, maximum likelihood estimates of three parameters, $t, c$, and $\pi$, were calculated. Maximum likelihood estimates of parameters in the finite mixture model were obtained by maximizing the log likelihood function:

$$
L(t, c, q, \pi, n, \boldsymbol{x})=\sum_{i=1}^{N} \ln \left[f\left(x_{i} ; t, c, q, \pi, n\right)\right],
$$

where $\boldsymbol{x}$ is the data vector, $t, c, q$, and $\pi$ are model parameters, $x_{i}$ is the number of errors committed by case $i$, and $N$ is the number of cases. Maximization is per- 
Table 1

Mixture Models That are Fit to the Data

\begin{tabular}{lccccc}
\hline \multicolumn{1}{c}{ Model } & $\begin{array}{c}\text { Incremental } \\
\text { Learners }\end{array}$ & $\begin{array}{c}\text { Rational } \\
\text { Learners }\end{array}$ & $\begin{array}{c}\text { Number of } \\
\text { Components }\end{array}$ & $\begin{array}{c}\text { Parameters } \\
\text { to Estimate }\end{array}$ & Designation \\
\hline $\operatorname{sol}(x ; .5, \alpha, 16)$ & + & - & 1 & $\alpha$ & sol \\
$\operatorname{ci}(x ; c, .5,16)$ & - & + & 1 & $c$ & $c i$ \\
$f(x ; t, c, .5, \pi, 16)$ & + & + & 2 & $t, c, \pi$ & $b-c i$ \\
$f(x ; .5, c, .5, \pi, 16)$ & + guess & + & 2 & $c, \pi$ & $b-c i^{*}$ \\
\hline
\end{tabular}

Note- $\mathrm{A}+$ means that the corresponding type of learners is modeled; - means that the corresponding type of learners is not modeled; guess means that the incremental learners respond at guessing level during the first 16 trials (i.e., $t$ is fixed at .5). We refer to these cases as slow learners.

formed by a quasi-Newton method (Titterington et al., 1985, p. 88). ${ }^{4}$ One at a time, $95 \%$ confidence intervals of the parameters estimates were estimated from the data by computing likelihood profiles (Meeker \& Escobar, 1995).

In addition to the two-component mixture model, the two single-component models are fitted to the data. Moreover, by fixing parameter $t$ to equal .5 , we tested whether slow learners responded by simply guessing during the first $n$ trials. In all, we fit four different models to each data set. Table 1 contains these models, which are denoted model sol (one-component single-operator linear model), $c i$ (one-component concept identification model), $b$-ci (two-component mixture model), and $b-c i^{*}$ (two-component mixture model with $t$ fixed at .5). Note that parameter $\pi$, the mixing proportion, is a free parameter in both two-component models.

4.4. Bayesian Posterior Probabilities of Component Membership. In fitting the two-component finite mixture distribution model, we did not need to determine the component membership of each subject (slow learner or a rational learner at a given occasion). The proportion of slow learners was determined by estimating the mixing proportion, $\pi$, without calculating component membership of each case. However, using the parameter estimates of the simplest, best fitting model, the posterior probability that a given case $i$ belongs to the binomial component (i.e., is a slow learner) can be calculated as follows:

$$
\begin{aligned}
\operatorname{Pr}[i \text { member of } b(x ; \hat{t}, n)] & \\
& =\frac{\hat{\pi} b\left(x_{i} ; \hat{t}, n\right)}{\hat{\pi} b\left(x_{i} ; \hat{t}, n\right)+(1-\hat{\pi}) \operatorname{ci}\left(x_{i} ; \hat{c}, q, n\right)} .
\end{aligned}
$$

Here $\hat{t}, \hat{c}$, and $\hat{\pi}$ are the maximum likelihood estimates of parameters $t, c$, and $\pi$ of a two-component model $f(x ; t$, $c, q, \pi, n)$, with $q$ and $n$ fixed at .5 and 16 , respectively. The variable $x_{i}$ represents the number of errors of case $i$. A given case $i$ can be assigned to the component with the highest posterior probability. Evidently, with an overlap of the components, not all posterior probabilities of individual cases will approach one or zero. In the case of intermediate probabilities, it is expected that some of the assignments will be wrong, which could influence the analysis based on these assignments. We can calculate the expected number of incorrect assignments given the data and a given model
E(\# wrong classifications)

$$
=\sum_{x=0}^{n} \frac{o_{x} \min [\hat{\pi} b(x ; \hat{t}, n),(1-\hat{\pi}) c i(x ; \hat{c}, q, n)]}{\hat{\pi} b(x ; \hat{t}, n)+(1-\hat{\pi}) c i(x ; \hat{c}, q, n)},
$$

where $o_{x}$ is the observed number of cases with $x$ errors and $\min [u, v]$ is $u$ if $u \leq v$ and is $v$ otherwise. Figure 1 shows an example of assigning cases to one of the components of the two-component mixture distribution $b$-ci model with $t=.5, c=.33$, and $\pi=.25$. In the Results section, we apply this method of assignment in order to study the consistency of learning behavior over measurement occasions.

\section{Goodness-of-Fit and Model Selection}

The models shown in Table 1 are fit to each data set. In each case, we wanted to select the model that fits best and is as simple as possible. Therefore, we needed both goodness-of-fit measures and a method to select between models that fit acceptably. There are a number of complicating issues involved in model fit and model selection of finite mixture distribution models, which we address below.

5.1. Goodness-of-Fit Statistics and Monte Carlo Bootstrap. By fitting a finite mixture distribution model to the data, we obtained expected frequencies. The goodness-of-fit of the model depends on the similarity between the expected frequencies and the observed frequencies. As a goodness-of-fit statistic, we calculate the $\log$ likelihood ratio statistic ${ }^{5}\left(\mathrm{G}^{2}\right.$; Read \& Cressie, $1988)$. The number of degrees of freedom $(d f)$ equals the number of cells minus 1 , minus the number of estimated parameters. The number of cells equals $n+1$ ( $n$ equals the number of trials that are analyzed; see Section 4). Mixture distribution model (Equation 4) with $n=16$, contains a high percentage of low frequencies (up to $94 \%$ of the cells contain fewer than five counts). A consequence of many small expected frequencies is that the theoretical $\chi^{2}$ distribution of the goodness-of-fit statistic does not hold, and so the $p$ value based on this theoretical distribution is incorrect (Greenwood \& Nikulin, 1996). Therefore, we apply a Monte Carlo bootstrap method to calculate reliable $p$ values. ${ }^{6}$ This method has been applied, for example, in latent class analysis of sparse data (Collins, Fidler, Wugalter, \& Long, 1993; Langeheine, Pannekoek, \& van de Pol, 1995; van der Heijden, 't Hart, \& Dessens, 1994) and to the likelihood ratio test statistic for 


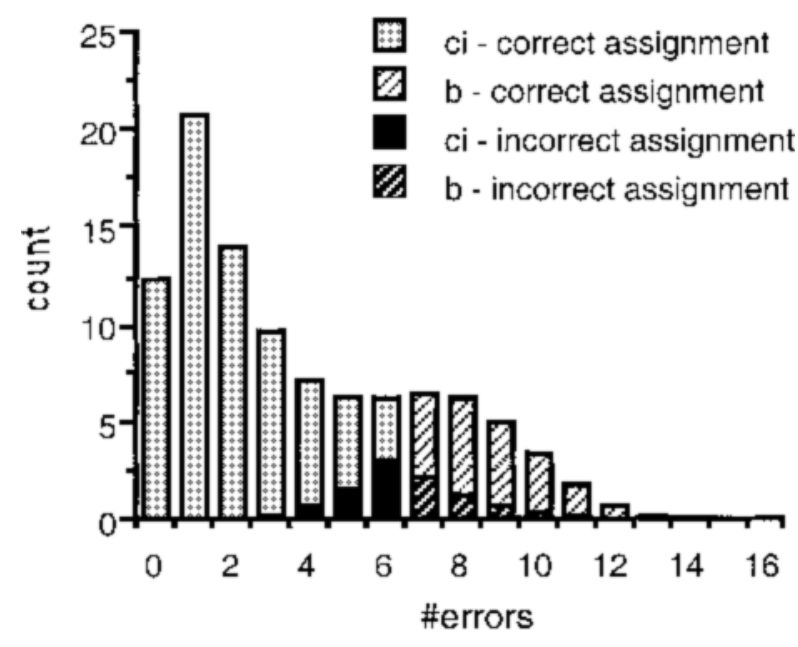

Figure 1. The correct and incorrect assignment of cases to a component of the mixture distribution. The distribution shown is that of a two-component $b$-ci $\operatorname{model}$ with $t=.5, c=.33$, and $\pi=$ .25. $c i=$ correct assignment $(b=$ correct assignment) means that cases are correctly assigned to the $c i$ component ( $b$ component); $c i=$ incorrect assignment $(b=$ incorrect assignment $)$ means that cases are assigned to the $c i$ component $(b=$ component) but belong to the $b$ component ( $c i$ component).

the number of components in a normal distribution (McLachlan, 1987).

5.2. Power Analysis. In the present approach to the analysis of the learning data, we based our conclusions about the experiments on a model that was found to fit the data. An appropriate model of the data yields expected frequencies that are similar to the observed frequencies. In cases where we wanted to base conclusions on the absence of differences, the power of the test was of special importance. Because the present sample sizes were not large, the power of some tests may be low. Low power would result in a high type II error. This implies that the probability of concluding that the model does not fit the data, given that the model is false in the population, would be small. Given the models we fit to the data, there is one important case in which this led to the wrong conclusion: A single component model (either a sol model or $c i$ model) may fit data that is actually derived from a two-component $b$-ci model. We examined the power of the goodness-of-fit tests of the models that we applied to the data by means of a simulation study. To this end, we simulated 250 data sets according to the two-component $b$-ci model with $t=.5, c=$ .33 , and $\pi=.25$. The one-component models sol (with parameter $\alpha$ estimated from the data) and $c i$ (with $c$ estimated from the data) were fit to each data set. Moreover, the $c i$ model was also fit to each data set with $c$ fixed at a particular value, once with $c=.33$ and once with $c=$ .5 , to show the effect in the power of constraining $c$ at a value that is expected a priori. We calculated the $\mathrm{G}^{2}$ and the corresponding $p$ value of each data set with the accompanying model. Finally, for each model, we counted the number of times it was correctly rejected at $\alpha=.05$. The results are shown in Figure 2.
The one-component sol model was correctly rejected in $100 \%$ of the cases, even when the number of cases $N$ was 30 . In contrast, the goodness-of-fit test of the onecomponent $c i$ model had very little power, unless $N=$ 1,000 . In this unconstrained model, the maximum likelihood estimate of $c$ lies between .1 and .2. The onecomponent $c i$ models with $c$ fixed at .33 and $c$ fixed at .5 were rejected much more often as models for the twocomponent data. Fixing parameter $c$ at plausible values of .33 or .5 increases the power considerably. Therefore, the maximum likelihood estimate of parameter $c$ in a onecomponent $c i$ model is of special importance. In the Results section, we will examine the confidence intervals of this parameter instead of fixing it.

5.3. Model Selection. In addition to calculating the goodness-of-fit of a model, we needed to make a choice between models that fit acceptably. If a parameter is fixed (e.g., we fix $t$ at .5 in a $b$-ci model to test whether slow learners respond at guessing level), the model is said to be nested under a model that includes this parameter as a free (to-be-estimated) parameter. The difference in fit of two hierarchically nested models can be tested by means of the log likelihood-difference statistic (minus twice the difference between the log likelihood of the constrained and the unconstrained model; Azzalini, 1996). Given the null hypothesis that the fixed parameter values are correct, the log likelihood-difference statistic is $\chi^{2}$-distributed, where the number of degrees of freedom equals the difference between the degrees of freedom of the two models.

On the contrary, the $s o l, c i$, and $b$-ci models are not hierarchically nested. In general, single-component models are not nested under multicomponent models (Everitt $\&$ Hand, 1981). To select between these models, we used the Akaike's information criterion (AIC). AIC equals minus twice the log likelihood plus twice the number of estimated parameters. Selection should favor the model with the lowest AIC. Since this measure takes into account the number of parameters estimated from the data, it discourages the selection of overly complex models.

\section{Results}

As mentioned, a total of 71 children participated in the experiment. The sample size varied slightly over the test occasions. The number of children who missed test occasions $\mathrm{B}, \mathrm{C}$, and $\mathrm{D}$, equaled 2,1 , and 3 , respectively. In addition, 8 cases did not satisfy the criterion of mastering the discrimination task within 100 trials. These cases ( 3 at occasion $\mathrm{A} ; 2$ at $\mathrm{B}$; and 3 at $\mathrm{C}$ ) were removed from the sample.

The presence of differences in the number of errors during the first 16 trials, between sexes, relevant stimulus dimensions (size vs. shape), age groups (6-, 7-, 8-, and 9year old subjects), and measurement occasions (A, B, C, and D) was investigated by means of nonparametric tests. Since we did not expect the number of errors to be normally distributed, we cannot apply normal theory parametric tests. Mann-Whitney U tests (corrected for ties) revealed no differences between the sexes at any of the 


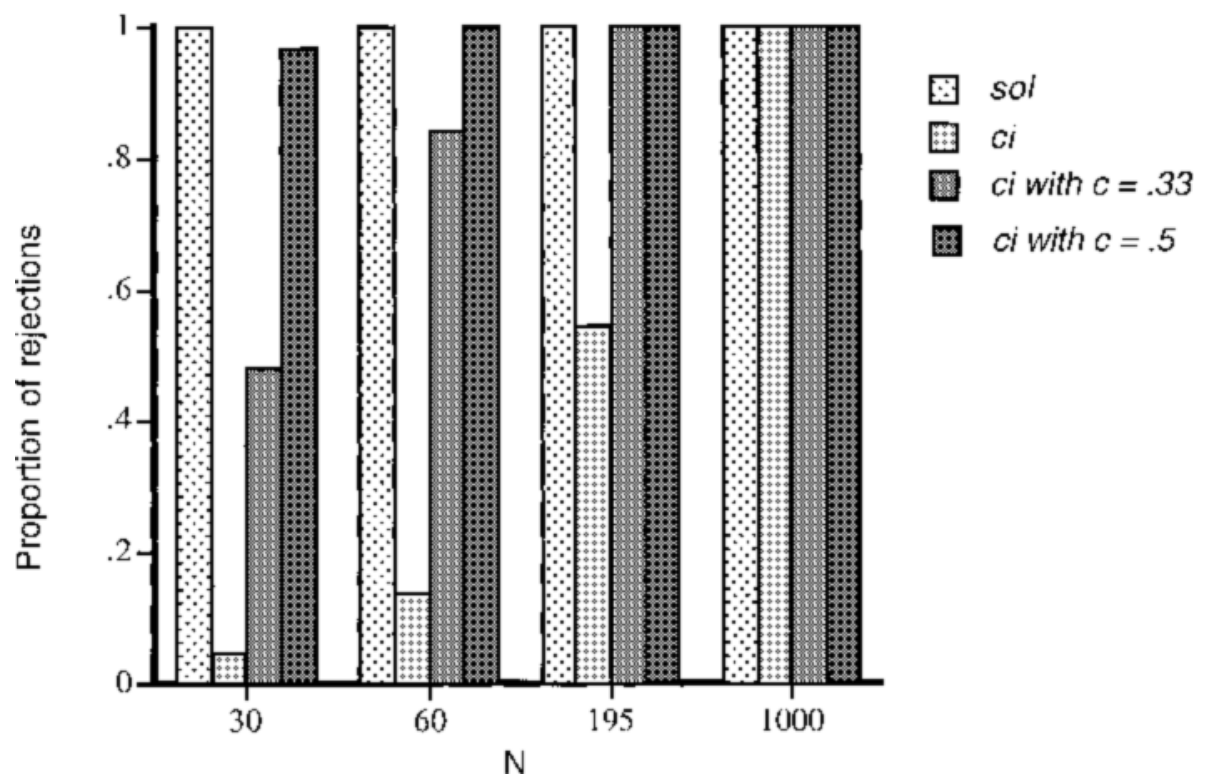

Figure 2. Results of the power analysis of goodness-of-fit tests. Two hundred fifty data sets are simulated according to a two-component $b$-ci model with $t=.5, c=.33$, and $\pi=.25$, and are modeled four times: by the sol model with parameter $\alpha$ estimated, by the $c i$ model with $c$ estimated, by the $c i$ model with $c$ fixed at .33 , and by the $c i$ model with $c$ fixed at .5 . The figure displays the proportion of times that the model is (correctly) rejected on the basis of the $\mathbf{G}^{2}$ statistic with significance level $\alpha=.05$.

measurements $(\mathrm{A}: \mathrm{Z}=-.81, p=.42 ; \mathrm{B}: \mathrm{Z}=-.80, p=.42$; $\mathrm{C}: \mathrm{Z}=-1.70, p=.09 ; \mathrm{D}: \mathrm{Z}=-.30, p=.76)$. Relevant stimulus dimensions, size and shape, were equally difficult at all of the measurement occasions $(\mathrm{A}: \mathrm{Z}=-.22, p=$ $.83 ; \mathrm{B}: \mathrm{Z}=-.24, p=.81 ; \mathrm{C}: \mathrm{Z}=-1.06, p=.29 ; \mathrm{D}: \mathrm{Z}=$ $-1.06, p=.29$ ). Hence, we pooled these groups, male and female, and the conditions, size and shape. Differences in the number of errors between measurements A, $\mathrm{B}$, and $\mathrm{C}$ were tested by means of Friedman's test and were found to be absent $\left[\chi^{2}(2)=3.84, p=.15\right.$, corrected for ties]. In contrast, there appeared to be differences between measurements $\mathrm{A}, \mathrm{B}, \mathrm{C}$, and $\mathrm{D}\left[\chi^{2}(3)=12.18, p=.007\right.$, corrected for ties]. Significant differences $(\alpha=.05 / 6=$ .008 with a Wilcoxon signed rank test) were limited to the comparison of occasions A and $\mathrm{D}(\mathrm{Z}=-3.94, p<.0001)$ and occasions $\mathrm{C}$ and $\mathrm{D}(\mathrm{Z}=-3.33, p=.0009)$; (A vs. $\mathrm{B}$ : $\mathrm{Z}=-2.13, p=.033$; A vs. $\mathrm{C}: \mathrm{Z}=-.08, p=.94$; $\mathrm{B}$ vs. $\mathrm{C}$ : $\mathrm{Z}=1.70, p=.09$; $\mathrm{B}$ vs. $\mathrm{D}: \mathrm{Z}=-.93, p=.35$ ). Age differences in the number of errors were investigated by comparing the 6-, 7-, 8-, and 9-year old subjects. These were found to be not significant for the data consisting of measurements A, B, and C [Kruskal-Wallis: $H(3)=7.75, p=$ $.052]$. We compared these preliminary results to the parameter estimates obtained by fitting the finite mixture distribution models to the data in Sections 6.2.1 and 6.2.2.

6.1. Statistical Tests for Markov Chains. In applying the distributions derived from the Markov sol model and ci model, and in applying the binomial distribution, we made various distinct assumptions about the learning processes. We adapted three tests from Suppes and Atkinson (1960) to test these assumptions. In these analyses, we include all cases that satisfied the criterion (i.e., mastered the task within 100 trials). For each case, we analyzed only trials observed before the 10 trials that comprised the criterion. Note that, in the finite mixture distribution analyses, we do not exclude trials that were part of the criterion run. For the trial sequences that were generated according to the Markov ci model, the present selection of trials means that only trials of the presolution state are analyzed. The number of analyzed trials varied over the cases, but the maximum number of analyzed trials per case equals 16 . These trials are the first 16 trials of each subject. In a number of cases, fewer than 16 trials were recorded because these subjects produced the set of 10 successive responses of which at least 9 were correct, (partly) before the 16th trial.

First, we assumed that the Markov chain for the selected trials is of order zero. Particularly in the sol model of incremental learners, this is a strong assumption. According to the sol model, successive trials of incremental learners are independent. According to the binomial model, successive trials of slow learners are also assumed to be independent during the first 16 trials. Moreover, the order is assumed to be zero for the rational learners in the presolution state, because they are assumed to respond at guessing level during the selected trials. Hence, for the selected trials of all cases, the order of the Markov chain is zero in the models that we apply. This hypothesis is acceptable $\left[\chi^{2}(1)=1.47, p=.23\right]$.

Second, we tested for stationarity of the first 16 trials. According to the $\mathrm{sol}$ model, response probabilities are not necessarily stationary, but may change as a function of 
trial number. However, if we fix the learning parameter $\alpha$ in the sol model to zero, this implies stationarity (i.e., a binomial distribution). This means that in the twocomponent model, we characterized slow learners by a constant probability of responding correctly during the first 16 trials. As it is quite feasible that incremental learners display substantial learning during the first 16 trials, this is a strong assumption. Moreover, we assume that the behavior of the rational learners was stationary during the presolution state, which was true according to the ci model. We tested the assumption of stationary probabilities by analyzing the mentioned response sequences of all cases. On the basis of the test of the assumption, it appears that the assumption of stationarity is tenable $\left[\chi^{2}(2)=4.07, p=.13\right]$.

Third, we assumed homogeneity of the response sequences across subjects. The assumption follows directly if the data can be modeled by a one-component model. But the two-component $b$-ci model also assumes homogeneity for the selected trials. Given that we only included the trials of each case before the criterion set (with a maximum of 16), we expected homogeneity of the answer sequences across subjects. Both rational learners in the presolution state (i.e., before they produced the required set of 10 responses) and slow learners were assumed to commit errors with a probability of .5, and the trials were assumed to be independent. It appears that the hypothesis of homogeneity is acceptable $\left[\chi^{2}(232)=\right.$ 238.91, $p=.36$ ].

To summarize, the three tests, relating to the order of the Markov chain, stationarity, and homogeneity, showed that the data agree with the assumptions underlying the sol model (with parameter $\alpha$ fixed at zero) as described by Equation 1, the $c i$ model as described by Equation 3 and the two-component $b$-ci mixture distribution model as described by Equation 4 .

6.2. Finite Mixtures Distribution Models. Again, all subjects who mastered the task within 100 trials were included in the analyses. As in the nonparametric analyses, the number of errors committed during the (at most) first 16 trials were analyzed. In contrast to the test of Markov chains, the responses on trials of the criterion run were included (if they occurred during the first 16 trials) to determine the number of errors. Two series of analyses were performed, one focusing on age group (pooling measurements $\mathrm{A}, \mathrm{B}$, and $\mathrm{C}$ ) and the other focusing on measurement occasions (pooling age groups 6 to 10). In each series, the models shown in Table 1 were fit to the datathat is, the one-component sol model, the one component $c i$ model, the two component $b$-ci model, and the twocomponent $b$-ci model with parameter $t$ fixed at $.5\left(b-c i^{*}\right.$ model).

6.2.1. Measurement occasions $A, B, C$, and Verbal Labeling. Table 2 contains the results for each measurement occasion. Measurement occasion D differs from the first three measurement occasions in that the subjects were required to supply verbal labels on the relevant stimulus dimension. It appears that at occasions A and B the one-component sol and $c i$ models do not fit the data, in contrast to the two-component $b$-ci model. At occasions $\mathrm{C}$ and $\mathrm{D}$, the one-component $c i$ model does fit the data (at $\mathrm{C}$, the estimated value of $c$ agrees with the power analysis, $\hat{c}=.16$ ). However, the AIC for the $b$-ci model is lowest for all four measurement occasions. Hence, at occasions $\mathrm{A}, \mathrm{B}, \mathrm{C}$, and $\mathrm{D}$, the $b$-ci model has the lowest AIC and is therefore judged to be the simplest, best fitting model for all measurement occasions. According to the 95\% confidence intervals, $\hat{c}$ in the $b$-ci models has values that were in the expected range- that is, between .33 and .5. According to the log likelihood difference statistic, fixing parameter $t$ of the $b$-ci model at .5 is accepted at occasions $\mathrm{C}$ and $\mathrm{D}$ but rejected at the $\alpha$ level of .05 at occasions $\mathrm{A}$ and $\mathrm{B}\left[\chi^{2}(1)=3.96, p=.047 ; \chi^{2}(1)=4.13, p=\right.$ .042 ; note that, at $\mathrm{A}$ and $\mathrm{B}, \hat{t}$ is larger than .5 , which would indicate that slow learners responded below guessing level]. However, since several (not completely independent) parallel tests were performed, an $\alpha$ level of .05 is relatively high. Hence, it seems safe to conclude that the slow learners responded at guessing level, but the issue will be of special concern in the analyses of age groups and in the analyses of the data of Experiment 2. Figure 3 shows the observed frequency distributions (bars) drawn together with the expected frequency distributions (lines) according the $b$-ci models with parameter $t$ fixed. The standardized residuals are shown in order to inspect the presence of patterns in deviations of the data from the models. Clear patterns are hard to discern.

Differences between occasions A, B, and C, on the one hand, and occasion $\mathrm{D}$, on the other hand, are observed only with respect to $\pi$, the proportion of slow learners. $\hat{\pi}$ is lowest at measurement occasion $\mathrm{D}$, which would indicate that the effect of verbal labeling is a decrease of the proportion of slow learners. A one-way test for two parameters with different variances and $N$ indicated that the mixing proportion (in the $b$ - $c i$ model with $t$ fixed) is significantly larger at $\mathrm{A}$ than at $\mathrm{D}(\alpha=.05 / 6 ; z=3.35, p<$ $.0004)$ and significantly larger at $\mathrm{C}$ than at $\mathrm{D}(z=2.49$, $p<.006) .{ }^{7}$ Some of the other pairwise comparisons approach significant differences (B vs. D: $z=1.47, p=.07$; A vs. B: $z=2.10, p=.018$; A vs. C: $z=.05, p=.31$ ). It appears that the results that were found in the nonparametric tests may be explained by differences in the proportions of slow learners.

6.2.2. Age groups $6,7,8$, and 9. The data of measurement occasions $\mathrm{A}, \mathrm{B}$, and $\mathrm{C}$ were pooled for the analyses within each age group. Because there were very few 10year-old subjects, they were removed from the analyses. To exclude the possibility that the two-component structure of the data was an artifact of the age structure of the sample, we first examined whether the two-component structure of the data was present within each age group. The analyses displayed in Table 3 show that this was the case. For all age groups, in contrast to the one-component models, the $b$-ci models fit the data well. Moreover, according to the AIC, the two-component $b$-ci model is the simplest, best fitting model. According to the $95 \%$ con- 
Table 2

Model Estimates for Measurement Occasions

\begin{tabular}{|c|c|c|c|c|c|c|c|c|c|c|c|c|}
\hline Model & $\hat{\alpha}$ & $\hat{t}$ & $\hat{c}$ & $\hat{\pi}$ & Logl. & $\mathrm{G}^{2}$ & $d f$ & $p\left(\mathrm{G}^{2}\right)$ & $\mathrm{AIC}$ & dlogl & $d f$ & $p(\mathrm{~d} \log 1)$ \\
\hline \multicolumn{13}{|c|}{ Measurement Occasion A, $N=68$} \\
\hline sol & $\begin{array}{c}.08 \\
(.07, .09)\end{array}$ & $\begin{array}{c}.50 \\
\text { fixed }\end{array}$ & & & -255.90 & 177.28 & 15 & .000 & 513.80 & & & \\
\hline$c i$ & & & $\begin{array}{c}.14 \\
(.11, .19)\end{array}$ & & -178.81 & 23.12 & 15 & .010 & 359.63 & & & \\
\hline$b-c i$ & & $\begin{array}{c}.58 \\
(.50, .70)\end{array}$ & $\begin{array}{c}.30 \\
(.18, .45)\end{array}$ & $\begin{array}{c}.27 \\
(.10, .45)\end{array}$ & -170.29 & 6.08 & 13 & .915 & 346.59 & & & \\
\hline$b-c i^{*}$ & & $\begin{array}{c}.50 \\
\text { fixed }\end{array}$ & $\begin{array}{c}.38 \\
(.24, .53)\end{array}$ & $\begin{array}{c}.38 \\
(.22, .50)\end{array}$ & -172.27 & 10.03 & 14 & .586 & 348.55 & 3.96 & 1 & .044 \\
\hline \multicolumn{13}{|c|}{ Measurement Occasion B, $N=67$} \\
\hline sol & $\begin{array}{c}0.14 \\
(.16, .12)\end{array}$ & $\begin{array}{c}.50 \\
\text { fixed }\end{array}$ & & & -232.72 & 196.8 & 15 & .000 & 467.44 & & & \\
\hline$c i$ & & & $\begin{array}{c}.25 \\
(.20, .31)\end{array}$ & & -155.48 & 42.30 & 15 & .000 & 312.96 & & & \\
\hline$b-c i$ & & $\begin{array}{c}.58 \\
(.50, .66)\end{array}$ & $\begin{array}{c}.49 \\
(.38, .60)\end{array}$ & $\begin{array}{c}.18 \\
(.10, .29)\end{array}$ & -141.13 & 13.60 & 13 & .333 & 288.25 & & & \\
\hline$b-c i^{*}$ & & $\begin{array}{c}.50 \\
\text { fixed }\end{array}$ & $\begin{array}{c}.51 \\
(.39, .63)\end{array}$ & $\begin{array}{c}.20 \\
(.11, .32)\end{array}$ & -143.19 & 17.73 & 14 & .113 & 290.38 & 4.13 & 1 & .040 \\
\hline \multicolumn{13}{|c|}{ Measurement Occasion C, $N=67$} \\
\hline sol & $\begin{array}{c}0.09 \\
(.07, .11)\end{array}$ & $\begin{array}{c}.50 \\
\text { fixed }\end{array}$ & & & -235.27 & 156.86 & 15 & .000 & 472.54 & & & \\
\hline$c i$ & & & $\begin{array}{c}.16 \\
(.12, .21)\end{array}$ & & -167.35 & 21.03 & 15 & .055 & 336.70 & & & \\
\hline$b-c i$ & & $\begin{array}{c}.55 \\
(.48, .65)\end{array}$ & $\begin{array}{c}.34 \\
(.21, .49)\end{array}$ & $\begin{array}{c}.28 \\
(.11, .44)\end{array}$ & -160.66 & 7.65 & 13 & .756 & 327.32 & & & \\
\hline$b-c i^{*}$ & & $\begin{array}{c}.50 \\
\text { fixed }\end{array}$ & $\begin{array}{c}.38 \\
(.25, .53)\end{array}$ & $\begin{array}{c}.33 \\
(.18, .47)\end{array}$ & -161.53 & 9.40 & 14 & .624 & 327.07 & 1.75 & 1 & .182 \\
\hline \multicolumn{13}{|c|}{ Measurement Occasion D, $N=68$} \\
\hline sol & $\begin{array}{c}0.19 \\
(.16, .22)\end{array}$ & $\begin{array}{c}.50 \\
\text { fixed }\end{array}$ & & & -170.12 & 96.18 & 15 & .000 & 342.24 & & & \\
\hline$c i$ & & & $\begin{array}{c}.34 \\
(.28, .42)\end{array}$ & & -131.00 & 17.94 & 15 & .043 & 264.00 & & & \\
\hline$b-c i$ & & $\begin{array}{c}.49 \\
(.37, .66)\end{array}$ & $\begin{array}{c}.47 \\
(.35, .61)\end{array}$ & $\begin{array}{c}.10 \\
(.02, .23)\end{array}$ & -127.56 & 11.05 & 13 & .337 & 261.11 & & & \\
\hline$b-c i^{*}$ & & $\begin{array}{c}.50 \\
\text { fixed }\end{array}$ & $\begin{array}{c}.47 \\
(.35, .59)\end{array}$ & $\begin{array}{c}.10 \\
(.02, .21)\end{array}$ & -127.58 & 11.10 & 14 & .468 & 259.16 & .05 & 1 & .807 \\
\hline
\end{tabular}

Note-Model sol is a one-component single-operator linear model; model $c i$ is a one-component $c i$ model; model $b$-ci is a two-component model with a binomial component and a $c i$ component; model $b$ - $c i^{*}$ is a two-component model with a binomial component and a $c i$ component with parameter $t$ fixed at $.5 ; \hat{\alpha}, \hat{t}, \hat{c}$, and $\pi$ are the maximum likelihood ( $\mathrm{ml}$ ) estimates of the parameters. The mixing proportion, $\pi$, of models $b$ - $c i$ and $b$ - $c i^{*}$ indicates the proportion of cases in the binomial component; the values in parentheses are the $95 \%$ confidence intervals of the mlestimates of the parameters above; Logl. is the log-likelihood of the model given the data; $\mathrm{G}^{2}$ is the likelihood-ratio statistic; $d f$ is the degrees of freedom; $p\left(\mathrm{G}^{2}\right)$ is the $p$ value of $\mathrm{G}^{2}$ obtained by Monte Carlo bootstrap; AIC is the Akaike's information criterion; d logl is the log-likelihood difference statistic; $p(\mathrm{~d} \log 1)$ is the $p$ value of dlogl with 1 degree of freedom.

fidence intervals, the estimates of parameter $c$ of the $b$-ci model lie within the expected range. According to the $\log$ likelihood difference statistic, fixing parameter $t$ at .5 is acceptable for all age groups, if we take into account that the $\alpha$ level of .05 is relatively high. Therefore, the slow learners of all age groups responded at guessing level during the first 16 trials.

Age-related differences in the estimates of parameter $\pi$, the proportion of slow learners, in the $b$-ci model with $t$ fixed, were analyzed. We tested for differences between 6 year olds ( $\tilde{\pi}=.42, S E=.097)$, on the one hand, and 7 year olds $(\hat{\pi}=.42, S E=.074 ; z=.93, p=.17), 8$ year olds $(\hat{\pi}=.18$, se $=.074 ; z=1.82, p=.034)$, and 9 year olds ( $\hat{\pi}=.21$, se $=.064 ; z=1.87, p=.031)$, on the other hand.
As expected, there were more slow learners among the 6 year olds than among the 8 -year-old and 9 -year-old subjects. However, because of the lack of power, differences were not completely convincing (significance level $\alpha=$ $.05 / 3$, since we made three pairwise comparisons - that is, the 6 year olds with the other age groups). No age-related differences were observed in the estimates of parameter $c$, the learning parameter of the rational learning mode.

6.3. Independence of Measurement Occasions. After fitting the mixture models to the data, each subject was assigned to a component by calculating the posterior probabilities according Equation 6. A case is simply assigned to the component associated with the largest posterior probability. Cases assigned to the $c i$ component were 

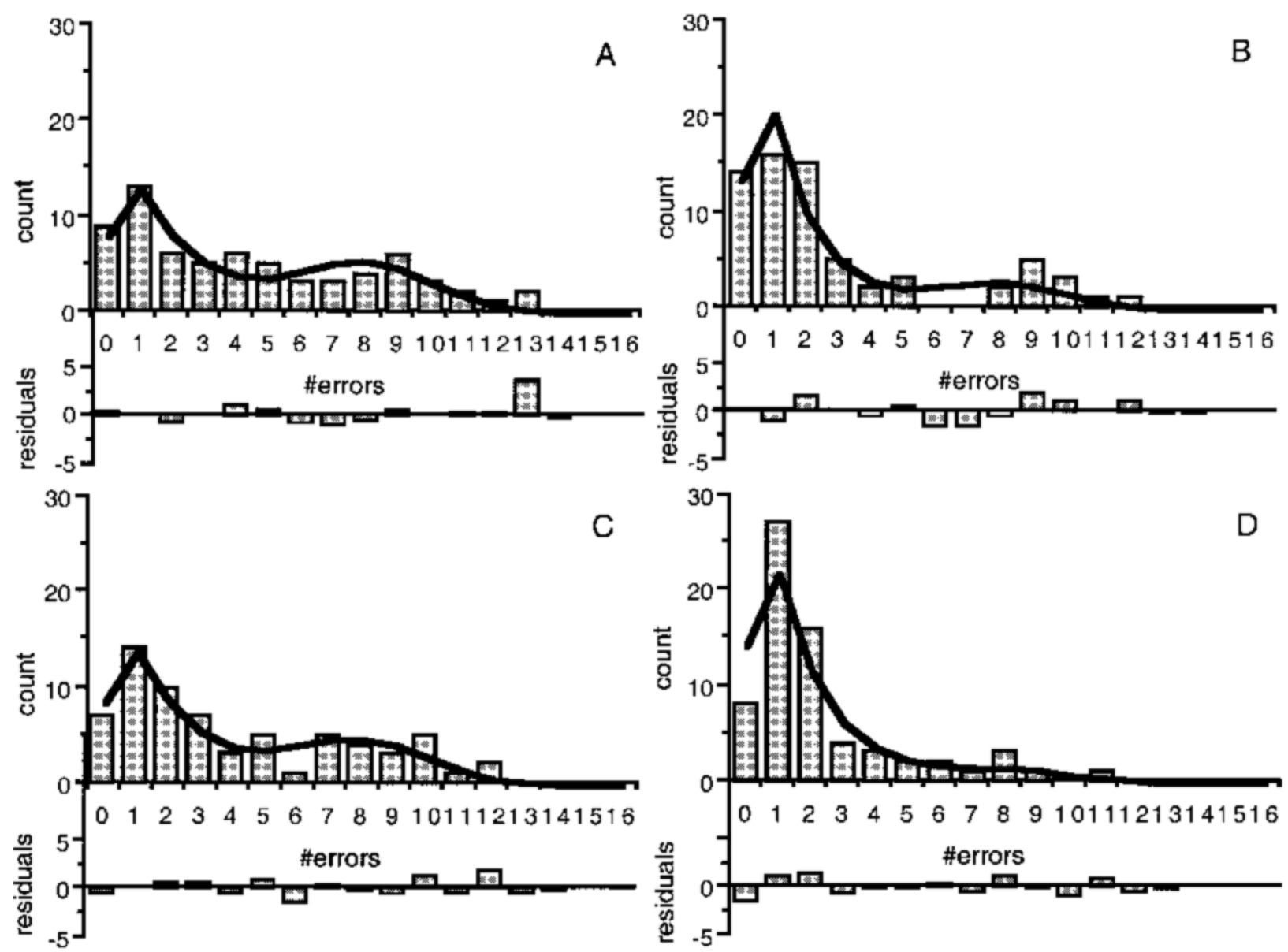

Figure 3. Frequency diagrams of observed number of errors (bars) and expected number of errors (lines) (i.e., according to the $b$ $c i$ model with $t$ fixed at .5) of the four different measurements. Occasion A: $\hat{c}=.33, \hat{\pi}=.38$; occasion B: $\hat{c}=.51, \hat{\pi}=.20$; occasion $\mathrm{C}: \hat{c}=$ $.38, \hat{\pi}=.33$; occasion $D$, the labeling condition: $\hat{c}=.47, \hat{\pi}=.10$. Below each diagram the standardized residuals, $\left(o_{x}-e_{x}\right) / \sqrt{e_{x}} 0 \leq x \leq$ 16, are plotted.

the rational learners (at the corresponding measurement occasion), cases assigned to the binomial component were the slow learners (at the corresponding measurement occasion). We base our mixture-model classification on the $b$-ci model with parameter $t$ fixed at .5. In assigning cases to either of the two components by their posterior probabilities, some cases, whose scores were in the area of overlap of the two component distributions, were classified incorrectly. The expected number of misclassifications according Equation 7 at occasion A equals six (four incorrectly assigned to the $b$ component and two to the $c i$ component). The total number of misclassifications at occasion $\mathrm{B}$ and $\mathrm{C}$ is two (two false $b$, zero false $c i$ ) and five (three false $b$, two false $c i$ ), respectively.

If children develop from purely slow learners into purely rational learners, the classifications at measurement occasions A, B, and $\mathrm{C}$ are expected to be dependent. In contrast, individuals may display a tendency to learn according to a certain mode. In that case, development could consist of an increase of the tendency to solve the discrimination rationally, as Kendler's (1995) theory posits. According to the latter hypothesis, the assignments should be independent between measurements, if no development takes place between the first and the last measurement occasion, which are about two weeks apart.

We found that the proportion of children responding according to one learning mode or the other did not vary significantly between measurement occasions A, B, and C. Hence, no learning effect between measurement occasions could be demonstrated. Next, it appeared that independence of measurement occasions could not be rejected for either pair of measurement occasions [A vs. B, $\chi^{2}(1)=.02, p=.89$; B vs. C, $\chi^{2}(1)=.91, p=.34 ; \mathrm{A}$ vs. $\left.\mathrm{C}, \chi^{2}(1)=1.60, p=.21\right]$. This confirms the hypothesis that children display a tendency to learn according to the rational mode, instead of responding consistently according to one mode. The mean tendency of an age group to learn rationally can be expressed as $1-\pi$ of the fitting $b$-ci model with fixed $t$ for that age group. 
Table 3

Model Estimates for Age Groups

\begin{tabular}{|c|c|c|c|c|c|c|c|c|c|c|c|c|}
\hline Model & $\hat{\alpha}$ & $\hat{t}$ & $\hat{c}$ & $\hat{\pi}$ & Logl. & $\mathrm{G}^{2}$ & $d f$ & $p\left(\mathrm{G}^{2}\right)$ & AIC & $\mathrm{d} \log 1$ & $d f$ & $p(\mathrm{~d} \log 1)$ \\
\hline \multicolumn{13}{|c|}{ Age Group $6, N=38$} \\
\hline sol & $\begin{array}{c}0.07 \\
(.05, .09)\end{array}$ & $\begin{array}{c}.50 \\
\text { fixed }\end{array}$ & & & -138.10 & 108.42 & 15 & .000 & 278.20 & & & \\
\hline$c i$ & & & $\begin{array}{c}.13 \\
(.08, .19)\end{array}$ & & -97.34 & 27.09 & 15 & .000 & 196.68 & & & \\
\hline$b-c i$ & & $\begin{array}{c}.56 \\
(.49, .64)\end{array}$ & $\begin{array}{c}.37 \\
(.20, .56)\end{array}$ & $\begin{array}{c}.39 \\
(.20, .57)\end{array}$ & -91.64 & 15.68 & 13 & .176 & 189.27 & & & \\
\hline$b-c i^{*}$ & & $\begin{array}{c}.50 \\
\text { fixed }\end{array}$ & $\begin{array}{c}.41 \\
(.22, .60)\end{array}$ & $\begin{array}{c}.42 \\
(.24, .60)\end{array}$ & -92.86 & 18.13 & 14 & .109 & 189.73 & 2.45 & 1 & .113 \\
\hline \multicolumn{13}{|c|}{ Age Group 7, $N=62$} \\
\hline $\begin{array}{l}\text { sol } \\
(.07, .11)\end{array}$ & $\begin{array}{c}0.09 \\
\text { fixed }\end{array}$ & .50 & & & -231.17 & 175.24 & 15 & .000 & 464.34 & & & \\
\hline$c i$ & & & $\begin{array}{c}.18 \\
(.13, .23)\end{array}$ & & -158.36 & 29.63 & 15 & .000 & 318.71 & & & \\
\hline$b-c i$ & & $\begin{array}{c}.59 \\
(.50, .69)\end{array}$ & $\begin{array}{c}.35 \\
(.23, .50)\end{array}$ & $\begin{array}{c}.24 \\
(.10, .39)\end{array}$ & -148.95 & 10.81 & 13 & .549 & 303.89 & & & \\
\hline$b-c i^{*}$ & & $\begin{array}{c}.50 \\
\text { fixed }\end{array}$ & $\begin{array}{c}.42 \\
(.28, .57)\end{array}$ & $\begin{array}{c}.31 \\
(.17, .46)\end{array}$ & -150.83 & 14.58 & 14 & .252 & 305.67 & 3.78 & 1 & .049 \\
\hline \multicolumn{13}{|c|}{ Age Group $8, N=43$} \\
\hline sol & $\begin{array}{c}0.11 \\
(.09, .13)\end{array}$ & $\begin{array}{c}.50 \\
\text { fixed }\end{array}$ & & & -139.88 & 93.18 & 15 & .000 & 281.76 & & & \\
\hline$c i$ & & & $\begin{array}{c}.21 \\
(.15, .28)\end{array}$ & & -103.73 & 20.86 & 15 & .047 & 209.45 & & & \\
\hline$b-c i$ & & $\begin{array}{c}.59 \\
(.44, .74)\end{array}$ & $\begin{array}{c}.30 \\
(.19, .45)\end{array}$ & $\begin{array}{c}.14 \\
(.01, .33)\end{array}$ & -101.26 & 15.93 & 13 & .170 & 208.52 & & & \\
\hline$b-c i^{*}$ & & $\begin{array}{c}.50 \\
\text { fixed }\end{array}$ & $\begin{array}{c}.33 \\
(.19, .48)\end{array}$ & $\begin{array}{c}.19 \\
(.00, .37)\end{array}$ & -101.92 & 17.24 & 14 & .122 & 207.83 & 1.31 & 1 & .251 \\
\hline \multicolumn{13}{|c|}{ Age Group $9, N=46$} \\
\hline sol & $\begin{array}{c}0.14 \\
(.12, .07)\end{array}$ & $\begin{array}{c}.50 \\
\text { fixed }\end{array}$ & & & -164.79 & 153.02 & 15 & .000 & 331.58 & & & \\
\hline$c i$ & & & $\begin{array}{c}.27 \\
(.20, .35)\end{array}$ & & -106.33 & 36.11 & 15 & .000 & 214.66 & & & \\
\hline$b-c i$ & & $\begin{array}{c}.57 \\
(.47, .69)\end{array}$ & $\begin{array}{c}.54 \\
(.37, .69)\end{array}$ & $\begin{array}{c}.18 \\
(.07, .33)\end{array}$ & -95.18 & 13.81 & 13 & .287 & 196.36 & & & \\
\hline$b-c i^{*}$ & & $\begin{array}{c}.50 \\
\text { fixed }\end{array}$ & $\begin{array}{c}.57 \\
(.41, .73)\end{array}$ & $\begin{array}{c}.21 \\
(.10, .35)\end{array}$ & -96.04 & 15.53 & 14 & .184 & 196.08 & 1.73 & 1 & .185 \\
\hline
\end{tabular}

Note-Symbols are explained in the note of Table 2.

\section{Cross-Validation of Mixture Modeling}

The data of a second experiment were subjected to the finite mixture distribution modeling to cross-validate the most important conclusion of the first experiment: the existence of two modes of simple discrimination learning.

7.1. Subjects. The sample consisted of 249 children from a primary school in The Netherlands in the age range of 4 to 12 years old and 26 adults, who were first year psychology students. The sample consisted of 107 girls and 85 boys.

7.2. Materials and Procedure. We assessed discrimination learning on a two-choice learning task. The task was similar to the task in the first experiment, apart from the dimensions of the stimuli that were varied (see Note 2). Stimuli differed again on two dimensions: shape (triangle/ square) and color (black/white). ${ }^{8}$ Stimuli were presented in pairs on a computer screen. The stimulus pairs were randomized in groups of four. The subjects responded by choosing either the left or the right stimulus by pressing a marked key on the left or the right of a keyboard. The in- struction and reinforcement were the same as in the first experiment. The process of learning to discriminate was continued until the criterion that 9 of the last 10 trials were correct was reached. The task comprised a maximum of 45 trials after which the test was interrupted. Each subject was tested once.

7.3. Results. Eighty-four subjects did not satisfy criterion within 45 trials and were removed from the sample. In the younger age groups, more individuals were removed than in the older age groups (4 to 6 years old: 53 out of 106; 7 to 9 years old: 24 out of $89 ; 10$ to 13 years old: 8 out of 55 ; none of the 26 students), which renders an analysis of age-related changes quite uninformative. Hence, we only analyze the data consisting of 192 subjects, to cross-validate the finite mixture distribution models that fit the data of the first experiment.

Table 4 summarizes the results of the model fits. The one-componentmodels, model sol and model $\mathrm{ci}$, do not fit the data. In contrast, the two-component model $b$ - $c i$ fits the data according to the $\mathrm{G}^{2}$ statistic and has the lowest 
Table 4

Model Estimates for Experiment 2

\begin{tabular}{|c|c|c|c|c|c|c|c|c|c|c|c|c|}
\hline Model & $\hat{\alpha}$ & $\hat{t}$ & $\hat{c}$ & $\pi$ & Logl. & $\mathrm{G}^{2}$ & $d f$ & $p\left(\mathrm{G}^{2}\right)$ & AIC & $\mathrm{d} \log 1$ & $d f$ & $p(\mathrm{~d} \log 1)$ \\
\hline sol & $\begin{array}{c}0.12 \\
(.11, .13)\end{array}$ & $\begin{array}{c}.50 \\
\text { fixed }\end{array}$ & & & -582.29 & 306.14 & 15 & .000 & 1166.58 & & & \\
\hline$c i$ & & & $\begin{array}{c}.22 \\
(.19, .25)\end{array}$ & & -441.11 & 23.78 & 15 & .000 & 884.21 & & & \\
\hline$b-c i$ & & $\begin{array}{c}.53 \\
(.46, .65)\end{array}$ & $\begin{array}{c}.32 \\
(.24, .41)\end{array}$ & $\begin{array}{c}.15 \\
(.04, .27)\end{array}$ & -434.36 & 10.29 & 13 & .429 & 874.73 & & & \\
\hline$b-c i^{*}$ & & $\begin{array}{c}.50 \\
\text { fixed }\end{array}$ & $\begin{array}{c}.34 \\
(.26, .41)\end{array}$ & $\begin{array}{c}.18 \\
(.09, .27)\end{array}$ & -434.57 & 10.70 & 14 & .557 & 873.14 & .21 & 1 & .651 \\
\hline
\end{tabular}

Note $-N=192$. Symbols are explained in the note of Table 2 .

AIC. Therefore, a two-component $b$ - $c i$ model appears to be the simplest, best-fitting model. The $95 \%$ confidence interval of parameter $c$, the learning parameter of rational learners, shows that $c$ is within the expected range of .33 and .5. We fixed parameter $t$, the probability of making an error by slow learners, at .5 to test the assumption that slow learners responded at guessing level during the first 16 trials. The log likelihood difference statistic shows that fixing $t$ at .5 does not result in a significant deterioration of fit of the model. Hence, it cannot be rejected that the slow learners responded at guessing level during the first 16 trials. It is noted that the larger number of subjects, 192 in the present experiment, in contrast to 68 in the first experiment, resulted in smaller $95 \%$ confidence intervals of the parameter estimates.

\section{Discussion}

Our application of finite mixture distribution modeling to cross-sectional data obtained in a simple discrimination task resulted in strong support for the existence of two distinct modes of learning behavior, as opposed to a single general mode of learning with a continuum of appearances. The analyses show that one-component models of either rational learners or incremental learners consistently fail to fit the data obtained in a simple discrimination task in the standard condition (i.e., without verbal labeling). All simplest, best-fitting models are twocomponent models comprising a concept-identification component and a binomial component. The conceptidentification component is a model for the behavior of rational learners. The binomial component is a model of no learning (within the first 16 trials). However, since all the subjects included in the analysis eventually mastered the task, we refer to the subjects whose behavior is modeled by the binomial component as slow learners. The two-component model also best fits the data of the second experiment. As shown, the two-component structure of the data is not an artifact of the age structure of the sample. Hence, the present results provide strong support for the hypothesis of two modes of learning in a simple discrimination task. In our opinion, the evidence for the existence of two modes of learning behavior is more convincing than that presented by Kendler (1979).

The maximum likelihood estimates of the model parameters, the learning parameter of the $c i$ component, the probability of the binomial component, and the mixing proportion, reveal the nature of the differences in performance between measurements. Although a single component model was found to fit the data obtained in the verbal labeling condition (occasion D), a two-component model fit the data considerably better. This implies that the two-component structure of the data was preserved given this experimental manipulation. Therefore, even if rational learning was encouraged, a small group of subjects persisted in learning slowly. According to nonparametric tests two out of three measurement occasions in the standard condition differed from the labeling condition. The model estimates show that this difference between standard condition and verbal labeling was due to the difference in the proportion of slow learners. That is, in the labeling condition, the proportion of slow learners was significantly smaller than it was at occasions A and C. However, occasion B did not differ from the labeling condition. Although this result is consistent with the outcomes of the nonparametric tests of differences in errors between measurement occasions, it is not clear why one of the measurement occasions in the standard condition did not differ significantly from the labeling condition. The most obvious reason is a lack of power to detect differences in the estimates of the mixing proportion. A second possibility, as Kendler (1995) reports, is that the probability of applying the rational learning mode may also be affected by motivational factors. An increasing number of rational learners by verbal labeling confirms Ashby et al.'s (1998) prediction that the categorization system that is actually applied by a subject in a categorization task can be manipulated experimentally.

Because of the lack of power, the evidence for the decreasing trend with age of the estimates of the proportion of slow learners is not completely convincing. An alternative way to test the equivalence of estimates of $\pi$ between age groups would be a multigroup analysis. In a multigroup analysis, equality constraints on parameters between models of different measurements can be tested directly. Concerning the estimates of parameter $c$ of the rational learning component, no age-related differences were observed. According to Ashby et al. (1998), rational learning is at a disadvantage in young children. With respect to the present learning task, we can conclude that if rational learning is applied, it is done so with equal effi- 
ciency by all age groups. The possible impairment of rational learning in young children may result in this learning's being applied less often in the present task.

By assigning subjects to either of the components of the mixture distribution model, we classified each subject at each measurement occasion as either a rational learner or a slow learner. It appears that the assignments at the first three measurement occasions were independent. Hence, we conclude that subjects did not consistently apply one learning mode. The results agree with Kendler's (1995) idea that children show a certain tendency to learn rationally, instead of displaying a definite and irrevocable transition from incremental to rational learning.

\section{Conclusion}

Individual differences in a cross-sectional group learning a simple discrimination task can be described as manifestations of two distinct modes of learning, as opposed to a continuum of manifestations of one learning mode. The first learning mode, rational learning, clearly involves focusing on rules. This learning mode fits nicely into Ashby et al.'s (1998) verbal categorization system. The second learning mode, slow learning, is a special case of the single-operator linear model, which is an incremental learning model. Incremental learning may involve focusing on items (cf. Kendler's nonselective encoding; Kendler, 1979) and is consistent with Ashby et al.'s implicit categorization system. However, it should be noted that the slow learning component of the two-component mixture model is not only a special case of the single-operator linear model, but of any kind of slow learning, including learning by small steps (Norman, 1972), but it is also a model of no learning. Nevertheless, we refer to the model as a model of slow learning because we only modeled subjects who eventually did master the task. The precise nature of the two learning processes underlying the two components of the distribution is open to debate. Here we applied only rudimentary models of rational learning and incremental learning. However, this discussion does not affect the conclusion about the twocomponent structure of the data, because the assumptions of the component distributions are satisfied, as the statistical tests for Markov chains show. Moreover, these tests indicate that more complex models, such as higher-order Markov models, are not warranted.

The issue of explicit learning versus implicit learning goes much farther than the question of whether a continuum of manifestations of a single learning mode exists. First, since the present study was a cross-sectional study of children's learning, it did not address the question of whether adults display two distinct learning modes. Indeed, the individual differences that we observed are not displayed by adults (Kendler, 1979). However, adults could possibly be encouraged to adopt incremental learning by suitable experimental manipulation. In that case, a continuous variation of independent variables, such as speed pressure or task load, would result in a twocomponent distribution of learning behavior (cf. Smith
\& Kemler, 1984). Second, the issue of explicit versus implicit learning concerns many other aspects of learning such as consciousness, verbalization, and robustness. It would be interesting to investigate whether the distinction that we found here can be related to these other properties of learning processes. Third, the existence of distinct modes of learning only provides partial information about the mechanism of development of learning simple discriminations. There are various explanations for the existence of distinct learning modes (e.g., Ashby et al., 1998). Below, we discuss this issue in the context of neural networks (i.e., connectionist models), which currently form the main paradigm for modeling learning mechanisms.

Among the researchers in the field of implicit learning, Reber (1993) argues that neural networks are appropriate to model implicit, but not explicit, learning. Nevertheless, several neural networks have been proposed that simulate learning behavior that is focused on rules. One of the aims of these neural network simulation studies is to model adult encoding behavior on discriminationshift tasks (i.e., set-shifting tasks or reversal-shift tasks; Kendler, 1995; Zeaman \& House, 1974a). This aim has been achieved partially (Bapi \& Denham, 1997; Kruschke, 1996; Raijmakers, van Koten, \& Molenaar, 1996; Sirois $\&$ Shultz, 1998). A second class of neural networks, which are referred to as models of frontal lobe functions, model hypothesis testing in learning simple discrimination tasks (e.g., Dehaene \& Changeux, 1991; D. S. Levine \& Prueitt, 1989). Although some of these studies have involved some modeling of child or impaired performance, the developmental process of discrimination learning has yet to be modeled in detail.

The outcome of the present study has important implications for neural network models of the development of discrimination learning. First, such models should display two modes of learning: slow learning and rational learning. Moreover, the developmental process is not a gradual change of the learning process. The two-component structure of the data implies that if the efficiency of the learning procedure increases with development, learning goes from some kind of slow learning to rational learning. It is not the case that, during development, inefficient learning gradually becomes more efficient until it is as efficient as rational learning. One possible model that may meet these demands is a modular system consisting of two distinct network modules: two neural modules (cf. Jacobs, Jordan, \& Barto, 1991), or a neural module combined with a production system (cf. Dehaene \& Changeux, 1991). A second possible neural network model includes a model parameter that through continuous variation, gives rise to two learning modes (Bapi \& Denham, 1997; D. S.Levine \& Prueitt, 1989; Sirois \& Shultz, 1998). Usually in neural networks, the gradual variation of a model parameter produces a gradual change in learning behavior. Here, unless the gradual change in parameter value is extremely rapid, this does not result in a two-component mixture distribution of the behavioral variable. However, in special cases, 
a gradual variation of a model parameter will induce a discontinuous change in behavior, which is called a bifurcation (e.g., Borisyuk \& Kirillov, 1992; Pollack, 1991; Raijmakers, van der Maas, \& Mohenaar, 1996). Such a discontinuous change will result in a two-component mixture distribution of the behavioral variable.

Although there may be many other unidentified mechanisms that give rise to distinct modes of learning, the empirical results presented here suggest that models of discrimination learning should take into account the occurrence of distinct learning modes during development.

\section{REFERENCES}

Ashby, F. G., Alfonso-Reese, L. A., Turken, A. U., \& Waldron, E. M. (1998) A neuropsychological theory of multiple systems in category learning. Psychological Review, 105, 442-481.

At kinson, R. C., Bower, G. H., \& Crothers, E. J. (1965). An introduction to mathematical learning theory (First corrected print, 1967). New York: Wiley.

AzZalini, A. (1996). Statistical inference based on the likelihood. London: Chapman \& Hall.

Bapi, R. S., \& Denham, M. J. (1997). Representational issues in neural systems: Example from a neural network model of set-shifting paradigm experiments. In J. A. Bullinaria, D. W. Glasspool, \& G. Houghton (Eds.), Fourth neural computation \& psychology workshop. London: Springer-Verlag.

Borisyuk, R. M., \& Kirillov, A. B. (1992). Bifurcation analysis of a neural network model. Biological Cybernetics, 66, 319-325.

Bower, G. H., \& Trabasso, T. (1964). Concept identification. In R. C. Atkinson (Ed.), Studies in mathematical psychology (pp. 32-94). Stanford, CA: Stanford University Press.

Collins, L. M., Fidler, P. L., Wugalter, S. E., \& Long, J. D. (1993). Goodness-of-fit testing for latent class models. Multivariate Behavioral Research, 28, 375-389.

Dehaene, S., \& Changeux, J. P. (1991). The Wisconsin card sorting test: Theoretical analysis and modeling in a neuronal network. Cerebral Cortex, 1, 62-79.

Dienes, Z, \& Berry, D. (1997). Implicit learning: Below the subjective threshold. Psychonomic Bulletin \& Review, 4, 3-23.

Dolan, C. V., \& VAN DER MAas, H. L. J. (1998). Fitting multivariate normal finite mixtures subject to constraints: Covariance and structure modeling. Psychometrika, 63, 227-253.

Estes, W. K. (1950). Toward a statistical theory of learning. Psychological Review, 57, 94-107.

Everitt, B. S., \& HAND, D. J. (1981). Finite mixture distributions. London: Chapman \& Hall.

Fischer, K. W., PiPP, S. L., \& Bullock, D. (1984). Detecting developmental discontinuities: Methods and measurement. In R. N. Emde \& R. J. Harmon (Eds.), Continuities and discontinuities in development (pp. 95-122). New York: Plenum.

Gohlson, B., Levine, M., \& Phillips, S. (1972). Hypotheses, strategies, and stereotypes in discrimination learning. Journal of Experimental Child Psychology, 13, 423-446.

Greenwood, P. E., \& NiKulin, M. S. (1996). A guide to chi-squared testing. New York: Wiley.

Heck, A. (1997). Introduction to Maple (2nd ed). New York: SpringerVerlag.

Hosenfeld, B., van der MaAs, H. L. J., \& VAN den Boom, D. C. (1997a). Indicators of discontinuous change in the development of analogical reasoning. Journal of Experimental Child Psychology, 64, 367-395.

Hosenfeld, B., van der MaAs, H. L. J., \& van den Boom, D. C. (1997b). Modeling frequency distributions of analogical reasoning performance from elementary school children. International Journal of Behavioral Development, 20, 529-547.

JacoBs, R. A., Jordan, M. I., \& Barto, A. G. (1991). Task decomposition through competition in a modular connectionist architecture: The what and where vision tasks. Cognitive Science, 15, 219-250.

JANSEN, B. R. J., \& VAN DER MAAS, H. L. J. (1997). A statistical test of the rule assessment methodology by latent class analysis. Developmental Review, 17, 321-357.

KendLER, T. S. (1979). The development of discrimination learning: A levels-of-functioning explanation. Advances in Child Development \& Behavior, 13, 83-117.

Kendler, T. S. (1995). Levels of cognitive development. Mahwah, NJ: Erlbaum.

Kruschke, J. K. (1996). Dimensional relevance shifts in category learning. Connection Science, 8, 225-247.

Langeheine, R, Pannekoek, J., \& VAN De Pol, F. (1995). Bootstrapping goodness-of-fit measures in categorical data analysis. The Netherlands: CBS statistics.

Levine, D. S., \& PrueitT, P. S. (1989). Modeling some effects of frontal lobe damage: Novelty and perseveration. Neural Networks, 2, 103-116.

Levine, M., Miller, P., \& Steynmeyer, C. H. (1967). The none-to-all theorem of human discrimination learning. Journal of Experimental Psychology, 73, 568-573.

McLachlan, G. J. (1987). On bootstrapping the likelihood ratio test statistic for the number of components in a normal distribution. Applied Statistics, 36, 318-324.

Meeker, W. Q., \& Escobar, L. A. (1995). Teaching about approximate confidence regions based on maximum likelihood estimation. The American Statistician, 49, 48-53.

Neal, A., \& HesKeth, B. (1997). Episodic knowledge and implicit learning. Psychonomic Bulletin \& Review, 4, 24-37.

Norman, M. F. (1972). Markov processes and learning models. New York: Academic Press.

Pollack, J. B. (1991). The induction of dynamical recognizers. Machine Learning, 7, 227-252.

Raijmakers, M. E. J., van der MaAs, H., \& Molenaar, P. C. M. (1996). Numerical bifurcation analysis of distance-dependent on-center offsurround shunting neural networks. Biological Cybernetics, 75, 495 507.

Raijmakers, M. E. J., van Koten, S., \& Molenaar, P. C. M. (1996). On the validity of simulating stagewise development by means of PDPnetworks: Application of catastrophe analysis and an experimental test for rule-like network performance. Cognitive Science, 20, 101-136.

ReAD, T. R. C., \& Cressie, N. A. C. (1988). Goodness-of-fit statistics for discrete multivariate data. New York: Springer-Verlag.

Reber, A. S. (1993). Implicit learning and tacit knowledge: An essay on the cognitive unconscious. New York: Oxford University Press.

REBer, A. S. (1997). How to distinguish implicit and explicit modes of acquisition. In J. D. Cohen \& J. W. Schooler (Eds.), Scientific approaches to consciousness. Carnegie Mellon on cognition (pp. 137159). Mahwah, NJ: Erlbaum.

Shanks, D. R., \& ST. John, M. F. (1994). Characteristics of dissociable human learning systems. Behavioral \& Brain Sciences, 17, 367-447.

SIEGLER, R. S. (1981). Developmental sequences within and between concepts. Monographs of the Society for Research in Child Development, 46, 1-74.

Silverman, I. W. (1966). Effects of verbalization on reversal shifts in children. Journal of Experimental Child Psychology, 4, 1-8.

Sirois, S., \& Shultz, T. R. (1998). Neural network modeling of developmental effects in discrimination shifts. Journal of Experimental Child Psychology, 71, 235-274.

Smith, J. D., \& Kemler, D. G. (1984). Overall similarity in adults' classification: The child in all of us. Journal of Experimental Psychology: General, 113, 137-159.

Stadler, M. A. (1997). Distinguishing implicit and explicit learning. Psychonomic Bulletin \& Review, 4, 56-62.

SuPPES, P., \& ATKInson, R. C. (1960). Markov learning models for multiperson interaction. Stanford, CA: Stanford University Press.

Thomas, H., \& Lohaus, A. (1993). Modeling growth and individual differences in spatial tasks. Monographs of the Society for Research in Child Development, 58, 1-169.

Thomas, H., \& Turner, G. (1991). Individual differences and development in the water-level task performance. Journal of Experimental Child Psychology, 51, 171-194.

Titterington, D. M., Smith, A. F. M., \& Makov, U. E. (1985). Statistical analysis of finite mixture distributions. Chichester: Wiley. Turner, C. W., \& Fischler, I. S. (1993). Speeded tests of implicit 
knowledge. Journal of Experimental Psychology: Learning, Memory, \& Cognition, 19, 1165-1177.

van der Heijden, P., 'T HART, H., \& Dessens, J. (1994). A parametric bootstrap procedure to perform statistical tests in latent class analysis. Kwantitatieve methoden, 17, 131-146.

van der MaAs, H. L. J., \& MolenaAr, P. C. M. (1992). Stagewise cognitive development: An application of catastrophe theory. Psychological Review, 99, 395-417.

Wohlwill, J. F. (1973). The study of development. New York: Academic Press.

Wolfe, J. H. (1970). Pattern clustering by multivariate mixture analysis. Multivariate Behavioral Research, 5, 329-350.

Zeaman, D., \& House, B. J. (1963). The role of attention in retardate discrimination learning. In N. R. Ellis (Ed.), Handbook of mental deficiency. New York: McGraw-Hill.

Zeaman, D., \& House, B. J. (1974a). Interpretation of developmental trends in discriminative transfer effects. In A. Pich (Ed.), Minnesota symposia on child psychology: Vol. 8. Minneapolis: University of Minnesota Press.

Zeaman, D., \& House, B. J. (1974b). A review of attention theory. In N. R. Ellis (Ed.), Handbook of mental deficiency, psychological theory \& research (2nd ed., pp. 63-120). New York: McGraw Hill.

\section{NOTES}

1. In terms of the Dutch school system, second year kindergarten corresponds to group 2 , fourth year primary school corresponds to group 6 .
2. The simple 2-choice discrimination learning task is presented as the first phase of a so-called optional-shift task. During the second and the third phase of this task, the reinforcement contingencies change. The precise design of phases two and three is not of interest here and is described in Kendler (1995).

3 . The sizes of the stimuli were chosen so that differences on the two dimensions were expected to be equally salient. The surface of the large stimuli is 8 in..$^{2}$, and the small stimuli are 4 in..$^{2}$, as in Silverman (1966).

4. We performed repeated optimization runs with different starting values to control for suboptimal solutions (local minima). Suboptimal solutions appeared to be rare.

5. The Pearson $\chi^{2}$ statistic is more sensitive to low expected model frequencies than is the log likelihood ratio statistic.

6 . We performed a small simulation study to test the application of the MC-bootstrap method to $\mathrm{G}^{2}$ of the finite mixture distribution model described by Equation 4. Mostly, the outcome of the bootstrapped $p$ values leads to the same correct conclusions concerning the acceptance or rejection of models at a $5 \%$ criterion as the original $p$ values. In general, the bootstrap $p$ values are a little bit lower than the theoretical $p$ values. In the Results section, we only show the bootstrap $p$ values.

7. To test the null hypothesis that $\pi_{1}=\pi_{2}$, we constructed the statistic $\left(\pi_{1}-\pi_{2}\right) /\left(\sigma_{1}^{2}+\sigma_{2}^{2}\right)^{1 / 2} . \pi_{1}$ and $\pi_{2}$ are the estimated mixture proportions. $\sigma_{1}^{2}$ and $\sigma_{2}^{2}$ are the variances of the parameter estimations. If $N>$ 30 for both groups, the statistic is approximately distributed according to a standard normal distribution (Thomas \& Turner, 1991).

8 . The sides of the squares and the equilateral triangles are $2 \mathrm{~cm}$. The stimuli are presented $5 \mathrm{~cm}$ left and right from the middle of the computer screen.

\section{APPENDIX \\ Probability Mass Function of the Number of Errors}

We adopt the Markov concept-identification (ci) model, which is described in the text (see Section 4.1), as a model of discrimination learning by rational learners. The $c i$ model is specified by the following transition matrix (Atkinson, Bower, \& Crothers, 1965):

$$
\begin{aligned}
& \text { State on trial } j+1 \\
& \text { (value of } F_{j+1} \text { ) }
\end{aligned}
$$

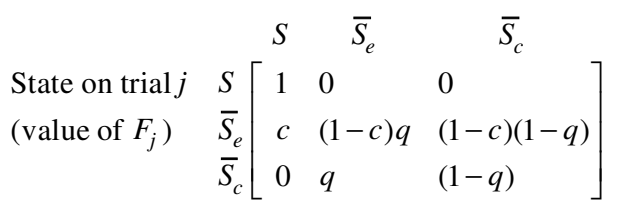

A subject $i$ can be in two states: the presolution state, $\bar{S}$, where an incorrect solution is given, and the solution state, $S$, where the correct solution is given. The presolution state is subdivided in two states: A subject in $\bar{S}_{e}$ is in the presolution state and generates an error; a subject in $\bar{S}_{c}$ is in the presolution state and responds correctly. $F_{j}$ is a random variable with three possible values: $S, \bar{S}_{e}, \bar{S}_{c}, \cdot c$ is the probability of selecting the correct hypothesis after committing an error, $q$ is the probability of an error in the presolution state-that is, $\operatorname{Pr}\left(F_{j}=\bar{S}_{e} \mid F_{j-1}=\bar{S}\right)$ - and $j$ is the trial number, $1 \leq j \leq n$. The matrix of the transition probabilities between the presolution state and the solution state defines a first order Markov process. The matrix of the transition probabilities of responding correctly and incorrectly in the presolution state alone defines a Bernoulli random process with independent trials.

Following Kendler (1995, p. 72), we add an assumption to the model

$$
\operatorname{Pr}\left(F_{1}=S\right)=\frac{c}{2} .
$$

That is, with a probability of one divided by the number of possible hypotheses about the discrimination rule, the subject starts in the solution state (see text, Section 4.1).

We are interested in the probability mass function of the number of errors committed in the first $n$ trials according to the $c i$ model. $T_{n}$ is the random variable of interest. For subject $i, T_{n}$ is defined as follows:

$$
T_{n}=\sum_{j=1}^{n} Y_{j,} Y_{j}=1 \text { if } F_{j}=\bar{S}_{e}, \quad Y_{j}=0 \text { otherwise. }
$$




\section{APPENDIX (Continued)}

Two situations are distinguished:either subject $i$ finds a solution or subject $i$ does not find a solution in $n$ trialsthat is, either $F_{n}=S$ or $F_{n}=\bar{S}$.

First, suppose $F_{n}=S$ :

If the solution is found without any errors, $T_{n}=0$, then $F_{1}=S$. Hence,

$$
\operatorname{Pr}\left(T_{n}=0\right)=\frac{c}{2}
$$

If a solution is found after making an error, $T_{n}>0$, then the following holds (a-e):

(a) The process started in the presolution state, $F_{1}=\bar{S}$

$$
\operatorname{Pr}\left(F_{1}=\bar{S}\right)=1-\frac{c}{2}
$$

(b) After the last error, a transition to the solution state is made. That is, and for some $F_{j-1}=\bar{S}_{e}$, and $F_{j}=$ $S$ for some $j, 2 \leq j \leq n$. The probability of the last error and a subsequent transition to the solution state equals

$$
\operatorname{Pr}\left(F_{j-1}=\bar{S}_{e} \wedge F_{j}=S \mid F_{j-2}=\bar{S}\right)=\operatorname{Pr}\left(F_{j-1}=\bar{S}_{e} \mid F_{j-2}=\bar{S}\right) \operatorname{Pr}\left(F_{j}=S \mid F_{j-1}=\bar{S}_{e}\right)=q c
$$

(c) If the total number of errors equals $x$, there are $x-1$ errors after which no transition to the solution state was made. The probability of one error without a transition to the solution state is

$$
\operatorname{Pr}\left(F_{j-1}=\bar{S}_{e} \wedge F_{j}=\bar{S} \mid F_{j-2}=\bar{S}\right)=\operatorname{Pr}\left(F_{j-1}=\bar{S} \mid F_{j-2}=\bar{S}\right) \operatorname{Pr}\left(F_{j}=\bar{S} \mid F_{j-1}=\bar{S}_{e}\right)=q(1-c) .
$$

The probability of $x-1$ errors without transition to the solution state is $[q(1-c)]^{x-1}$.

(d) If the last error is at trial $j$, then there are $x-1$ errors during the first $j-1$ trials, $x \leq j<\mathrm{n}$. The number of different sequences of $(x-1)$ errors in $j-1$ trials is

$$
\left(\begin{array}{l}
j-1 \\
x-1
\end{array}\right)
$$

(e) The probability of a correct response in the presolution state and staying in the presolution state,

$$
\operatorname{Pr}\left(F_{j-1}=\bar{S}_{c} \wedge F_{j}=\bar{S} \mid F_{j-2}=\bar{S}\right)=\operatorname{Pr}\left(F_{j-1}=\bar{S}_{c} \mid F_{j-2}=\bar{S}\right) \operatorname{Pr}\left(F_{j}=\bar{S} \mid F_{j-1}=\bar{S}_{c}\right)=1-q .
$$

Now it follows directly that if $F_{n}=S$, then for $1<x \leq n$

$$
\operatorname{Pr}\left(T_{n}=x\right)=\left(1-\frac{c}{2}\right) q c[q(1-c)]^{x-1} \sum_{j=x}^{n}\left(\begin{array}{c}
j-1 \\
x-1
\end{array}\right)(1-q)^{j-x} .
$$

Unfortunately, the summation of Expression A2 cannot be simplified any further.

Next, suppose $F_{n}=\bar{S}$. That is, after $n$ trials no solution is found. Then the following holds (a-b):

(a) Since no transition from the solution state to the presolution state is possible, the process starts in the presolution state.

$$
\operatorname{Pr}\left(F_{1}=\bar{S}\right)=1-\frac{c}{2}
$$

(b) During $n$ trials, $x$ trials are errors and $n-x$ trials are correct. The probability of committing an error in the presolution state and staying in the presolution state is $q(1-c)$. The probability of responding correctly in the presolution state and staying in the presolution state is $(1-q)$.

Hence, for $0 \leq x \leq n$.

$$
\operatorname{Pr}\left(T_{n}=x\right)=\left(1-\frac{c}{2}\right)\left(\frac{n}{x}\right)[q(1-c)]^{x}(1-q)^{n-x} .
$$

From this it follows that

$$
\operatorname{Pr}\left(T_{n}=0\right)=\left(1-\frac{c}{2}\right)(1-q)^{n}
$$


Finally, combining Equations A1, A2, A3, and A4 results in the probability mass function of $T_{n}$

$$
\begin{aligned}
& \operatorname{Pr}\left(T_{n}=0\right)=\frac{c}{2}+\left(1-\frac{c}{2}\right)(1-q)^{n} \\
& \operatorname{Pr}\left(T_{n}=x\right)=\left(1-\frac{c}{2}\right) q c[q(1-c)]^{x-1} \sum_{j=x}^{n}\left(\begin{array}{c}
j-1 \\
x-1
\end{array}\right)(1-q)^{j-x}+\left(1-\frac{c}{2}\right)\left(\begin{array}{l}
n \\
x
\end{array}\right)[q(1-c)]^{x}(1-q)^{n-x} \\
& 1 \leq x \leq n .
\end{aligned}
$$

(Manuscript received January 20, 1999;

revision accepted for publication January 11,2001.) 